\title{
Rigorous derivation of the Kuramoto-Sivashinsky equation from a 2D weakly nonlinear Stefan problem
}

\author{
Claude-Michel Brauner \\ Institut de Mathématiques de Bordeaux, Université de Bordeaux, \\ 33405 Talence Cedex, France \\ E-mail: claude-michel.brauner@u-bordeauxl.fr \\ JOSEPHUS HuLSHOF \\ Faculty of Sciences, Mathematics and Computer Sciences Division, \\ VU University Amsterdam, 1081 HV Amsterdam, The Netherlands \\ E-mail: jhulshof@cs.vu.nl
}

LUCA LORENZI

Dipartimento di Matematica, Università di Parma, Parco Area delle Scienze 53/A, 43124 Parma, Italy

E-mail: luca.lorenzi@uniprit

[Received 14 July 2009 and in revised form 16 July 2010]

\begin{abstract}
We are interested in a rigorous derivation of the Kuramoto-Sivashinsky (K-S) equation from a free boundary problem. As a paradigm, we consider a two-dimensional Stefan problem in a strip, a simplified version of a solid-liquid interface model. Near the instability threshold, we introduce a small parameter $\varepsilon$ and define rescaled variables accordingly. At fixed $\varepsilon$, our method is based on: definition of a suitable linear 1D operator, projection with respect to the longitudinal coordinate only, and the Lyapunov-Schmidt method. As a solvability condition, we derive a self-consistent parabolic equation for the front. We prove that, starting from the same configuration, the latter remains close to the solution of K-S on a fixed time interval, uniformly in $\varepsilon$ sufficiently small.
\end{abstract}

2010 Mathematics Subject Classification: Primary 35K55; Secondary 35R35, 35B25, 80A22.

Keywords: Kuramoto-Sivashinsky equation; front dynamics; Stefan problem; singular perturbations; pseudo-differential operators.

\section{Introduction}

A very challenging problem in free boundary problems is the derivation of a single equation for the interface or moving front which captures the dynamics of the system, at least asymptotically, when a suitable parameter $\varepsilon$ tends to 0 . The Kuramoto-Sivashinsky equation, which we abbreviate hereafter as the K-S equation, or simply K-S,

$$
\Phi_{\tau}+\nu \Phi_{\eta \eta \eta \eta}+\Phi_{\eta \eta}+\frac{1}{2}\left(\Phi_{\eta}\right)^{2}=0
$$

appears in a variety of domains in physics and chemistry, where it models cellular instabilities, pattern formation, turbulence phenomena and transition to chaos. Among many references, we refer to the pioneering papers [12, 10]. The former was historically the first asymptotical derivation of 
K-S, in the framework of the Near Equidiffusive Flames model, in short NEF, in combustion theory. The latter provided the first comprehensive computational study. We also refer the reader to the book [14] and its extensive bibliography. Our purpose here is to provide some rigorous mathematical commentary on the derivation of this well-known model from a two-dimensional Stefan problem. We point out that our method might work for other systems allowing scale separation and thereby reduction of the effective dimensionality of the problem. In this respect, we have considered the NEF problem in [5, 6].

As one would surmise at the outset, the K-S model involves a balance between several effects. Roughly speaking, K-S arises when the competing effects of a destabilizing linear part and a stabilizing nonlinearity are the dominant processes in physical reality. The linear instability is itself the result of a competition between two linear operators, $\mathcal{A}=D_{\eta \eta}$ and $\nu \mathcal{A}^{2}$ (we call $\nu \mathcal{A}^{2}+\mathcal{A}$ the Kuramoto-Sivashinsky linear operator).

Put another way, the K-S equation is the simplest, and indeed a paradigm system in which these effects compete equally. It is this dominant balance that is explored rigorously in the present essay. It will turn out that, in deriving K-S as an asymptotic limit of more complex systems, only certain type of terms contribute to the lowest order of approximation. Other types of terms will lead to higher order perturbations. In a forthcoming paper, we intend to consider the effects of these higher order perturbations on the basic K-S system.

As a paradigm two-dimensional problem (see [3, 2, 1] for the one-dimensional case and the Q-S equation in flame front dynamics), we consider a solid-liquid interface model introduced by Frankel in [8]. The solidification front is represented by $x=\xi(t, y)$. The liquid phase occurs when $x<$ $\xi(t, y)$, the solid one when $x>\xi(t, y)$. The dynamics of heat is described by the heat conduction equation

$$
T_{t}(t, x, y)=\Delta T(t, x, y), \quad x \neq \xi(t, y),
$$

where $y \in[-\ell / 2, \ell / 2]$ with periodic boundary conditions. At $-\infty$, the temperature of the liquid is normalized to 0 . At the front $x=\xi(t, y)$ there are two conditions. First, the balance of energy at the interface is given by the jump

$$
\left[\frac{\partial T}{\partial n}\right]=V_{n},
$$

where $V_{n}$ is the normal velocity. Second, according to the Gibbs-Thompson law, the nonequilibrium interface temperature is defined by

$$
T=1-\gamma \kappa+r\left(V_{n}\right),
$$

where the melting temperature has been normalized to $1, \kappa$ is the interface curvature and the positive constant $\gamma$ represents the solid-liquid surface tension. The function $r$ is increasing and such that $r(-1)=0, r^{\prime}(-1)=1$ (see [8, 9]). Hereafter, we assume that $r-1$ is linear and we replace the curvature by the second-order derivative. Therefore, 1.4 becomes

$$
T=1-\gamma \xi_{y y}+V_{n}+1 .
$$

It is not difficult to see that system (1.2), (1.3), (1.4) admits a one-phase planar travelling wave (TW) solution $(T(t, x), \xi(t))=(\Theta(t+x),-t)$, where $\Theta(z)=e^{z}$ for $z<0$, and $\Theta(z)=1$ for $z>0$.

As usual, we fix the free boundary. We set $\xi(t, y)=-t+\varphi(t, y), x^{\prime}=x-\xi(t, y)$ and we will omit primes. In this new framework, 1.2 reads

$$
T_{t}+\left(1-\varphi_{t}\right) T_{x}=\Delta_{\varphi} T, \quad x \neq 0,
$$


where $\Delta_{\varphi}=\left(1+\left(\varphi_{y}\right)^{2}\right) D_{x x}+D_{y y}-\varphi_{y y} D_{x}-2 \varphi_{y} D_{x y}$. The front is now fixed at $x=0$. The first condition (1.3) reads

$$
\varphi_{t}=1+\left(1+\left(\varphi_{y}\right)^{2}\right)\left[T_{x}\right]
$$

whereas we replace $(1.5)$ by

$$
T=1-\gamma \varphi_{y y}+\varphi_{t}+\frac{1}{2}\left(\varphi_{y}\right)^{2} .
$$

Introducing the temperature perturbation $u=T-\Theta$, the problem for the couple $(u, \varphi)$ reads

$$
u_{t}+\left(1-\varphi_{t}\right) u_{x}-\Delta_{\varphi} u-\varphi_{t} \Theta_{x}=\left(\Delta_{\varphi}-\Delta\right) \Theta, \quad x \neq 0,
$$

where

$$
\left(\Delta_{\varphi}-\Delta\right) \Theta=\left\{\left(\varphi_{y}\right)^{2}-\varphi_{y y}\right\} e^{x} \chi(-\infty, 0)=\left(\left(\varphi_{y}\right)^{2}-\varphi_{y y}\right) \Theta_{x} .
$$

As in [4], we make further simplifications: (i) we consider a quasi-steady problem, dropping the time derivative $u_{t}$ in 1.77; (ii) we take a linearized problem for $u$; (iii) we limit ourselves to considering only the second-order terms in the jump conditions at $x=0$. Actually, as observed in similar problems (see [3]), not far from the instability threshold the time derivative in the temperature equation has a relatively small effect on the solution. Our final system reads

$$
\begin{aligned}
& u_{x}-\Delta u-\varphi_{t} \Theta_{x}=\left(\Delta_{\varphi}-\Delta\right) \Theta, \quad x \neq 0, \\
& \varphi_{t}=\left[u_{x}\right]-\left(\varphi_{y}\right)^{2}, \\
& u_{\mid x=0}=-\gamma \varphi_{y y}+\varphi_{t}+\frac{1}{2}\left(\varphi_{y}\right)^{2} .
\end{aligned}
$$

For the convenience of the reader, we recall the main results of [4], where we considered problem (1.8)-(1.10) in the strip $\mathbb{R} \times[-\ell / 2, \ell / 2]$, with periodic boundary conditions prescribed at $y= \pm \ell / 2$. More precisely, we studied the stability of the TW solution and proved the following result: there exists $\gamma_{c}<1$ such that

(i) for $\gamma>\gamma_{c}$, the TW solution to problem [1.8)-(1.10) is orbitally stable (with asymptotic phase);

(ii) for $0<\gamma<\gamma_{c}$, the TW is unstable.

We also showed that $\gamma_{c}=1-3 \lambda_{1}(\ell)+\cdots$, where $-\lambda_{1}(\ell)=-4 \pi^{2} / \ell^{2}$ is the largest eigenvalue of the realization of $D_{y y}$ in $C([-\ell / 2, \ell / 2])$ with periodic boundary conditions and zero average.

The main tool is the derivation of a self-consistent equation for the front $\varphi$ :

$$
\varphi_{t}+G\left(\left(\varphi_{y}\right)^{2}\right)=\Omega \varphi, \quad|y| \leqslant \ell / 2,
$$

where both $\Omega$ and $G$ are linear pseudo-differential operators whose symbols $\omega_{k}$ and $g_{k}$ are explicit and $g_{0}=1 / 2$, which is reminiscent of K-S. If we think formally of (1.11) in the whole space (i.e. $\ell=+\infty)$, then $\omega_{k}$ is the growth rate which expands, for small wave number $k$, as

$$
\omega_{k}=(1-\gamma) k^{2}+(\gamma-4) k^{4}+\cdots,
$$

with exchange of stability at $\gamma=1$. Therefore, when $\gamma$ is close to unity, but smaller, it is natural to introduce a small parameter $\varepsilon>0$, setting

$$
\gamma=1-\varepsilon,
$$


and define the rescaled dependent and independent variables accordingly:

$$
t=\tau / \varepsilon^{2}, \quad y=\eta / \sqrt{\varepsilon}, \quad u=\varepsilon^{2} v, \quad \varphi=\varepsilon \psi .
$$

Then we anticipate, in the limit $\varepsilon \rightarrow 0$, that $\psi \simeq \Phi$, where $\Phi$ solves the following K-S equation (with $v=3$ ):

$$
\Phi_{\tau}+3 \Phi_{\eta \eta \eta \eta}+\Phi_{\eta \eta}+\frac{1}{2}\left(\Phi_{\eta}\right)^{2}=0
$$

This is what we have to establish in a rigorous mathematical way. Let us fix $\ell_{0}>0$. The main idea is to link the small parameter $\varepsilon$ and the width of the strip, which will become larger and larger as $\varepsilon \rightarrow 0$, i.e., as $\gamma \rightarrow 1$. Take for $\ell$ the quantity

$$
\ell_{\varepsilon}=\ell_{0} / \sqrt{\varepsilon}
$$

which blows up as $\varepsilon \rightarrow 0$ and the strip $\mathbb{R} \times\left[-\ell_{\varepsilon} / 2, \ell_{\varepsilon} / 2\right]$ approaches $\mathbb{R}^{2}$. We easily see that $\lambda_{1}\left(\ell_{\varepsilon}\right)=4 \pi^{2} / \ell_{\varepsilon}^{2}=4 \pi^{2} \varepsilon / \ell_{0}^{2}$; hence,

$$
\gamma_{c}=1-\frac{12 \pi^{2}}{\ell_{0}^{2}} \varepsilon+\ldots
$$

Thus, $\ell_{0}$ becomes the new bifurcation parameter. We shall assume that $\ell_{0}>\sqrt{12} \pi$ in order to have $\gamma_{c} \in(1-\varepsilon, 1)$, i.e., $\gamma>\gamma_{c}$, otherwise the TW is stable and the dynamics is trivial. Clearly, this is related to the stability of the null solution to K-S. The relevant eigenvalue of the KuramotoSivashinsky linear operator $3 \mathcal{A}^{2}+\mathcal{A}$ is $3\left(\lambda_{1}\left(\ell_{0}\right)\right)^{2}-\lambda_{1}\left(\ell_{0}\right)$ which vanishes for $\lambda_{1}\left(\ell_{0}\right)=1 / 3$, i.e., when $\ell_{0}=\sqrt{12} \pi$.

An important feature of this paper is that we work in the fixed strip $\mathbb{R} \times\left[-\ell_{0} / 2, \ell_{0} / 2\right]$, with the rescaled variables 1.12 . We will return to the original variable only in the final section.

The main result is the following.

MAIN THEOREM 1.1 Fix $\alpha \in(0,1 / 2)$ and let $\Phi_{0} \in C^{6+2 \alpha}\left(\left[-\ell_{0} / 2, \ell_{0} / 2\right]\right)$ satisfy the condition $D_{\eta}^{(k)} \Phi_{0}\left(-\ell_{0} / 2\right)=D_{\eta}^{(k)} \Phi_{0}\left(\ell_{0} / 2\right)$ for any $k=0, \ldots, 6$. Let $\Phi$ be the periodic solution of 1.13 (with period $\ell_{0}$ ) on a fixed time interval $[0, T]$, satisfying the initial condition $\Phi(0, \cdot)=\Phi_{0}$. Then there exists $\varepsilon_{0}=\varepsilon_{0}(T) \in(0,1 / 2)$ such that, for $0<\varepsilon \leqslant \varepsilon_{0}$, problem (1.8)-1.10) admits a unique smooth solution $(u, \varphi)$ on $\left[0, T / \varepsilon^{2}\right]$, which is periodic with period $\ell_{0} / \sqrt{\varepsilon}$ with respect to $y$, and satisfies

$$
\varphi(0, y)=\varepsilon \Phi_{0}(y \sqrt{\varepsilon}), \quad|y| \leqslant \frac{\ell_{0}}{2 \sqrt{\varepsilon}} .
$$

Moreover, there exists a positive constant $C$, independent of $\varepsilon \in\left(0, \varepsilon_{0}\right]$, such that

$$
\left|\varphi(t, y)-\varepsilon \Phi\left(t \varepsilon^{2}, y \sqrt{\varepsilon}\right)\right| \leqslant C \varepsilon^{2}, \quad 0 \leqslant t \leqslant \frac{T}{\varepsilon^{2}},|y| \leqslant \frac{\ell_{0}}{2 \sqrt{\varepsilon}} .
$$

For a precise definition of what smooth solution means we refer the reader to Subsection 6.3

Clearly, the initial condition for $\varphi$ is of special type, compatible with $\Phi_{0}$ and (1.1) at $\tau=0$. Initial conditions of this type have already been considered in [1, 3].

The paper is organized as follows. In Section 2 we introduce some notation and the function spaces we extensively use throughout the paper. In Section 3 we proceed to a formal Ansatz in the 
spirit of [12]. We set $\gamma=1-\varepsilon$, expand $v=v^{0}+\varepsilon v^{1}+\cdots, \psi=\psi^{0}+\varepsilon \psi^{1}+\cdots$, and show that $\psi^{0}$ satisfies the K-S equation (1.13), thanks to an elementary solvability condition. The paper consists in giving a rigorous proof of the Ansatz (i.e., of Main Theorem 1.1], thanks to an abstract solvability condition within the framework of adequate function spaces. In this respect, in Section 4 we transform system (1.8)-(1.10) in an equivalent problem (for the new unknowns) using the techniques of [4], which are based on

(i) definition of a suitable linear one-dimensional operator;

(ii) projection with respect to the $x$ coordinate only;

(iii) Lyapunov-Schmidt method.

This allows us to decouple the system into a self-consistent fourth-order (in space) parabolic equation for the front $\psi$ and an elliptic equation which can be easily solved whenever a solution to the front equation is determined. Hence, the rest of the paper is devoted to the parabolic equation. In this direction, according to the Ansatz, we split $\psi=\Phi+\varepsilon \rho_{\varepsilon}$. In Section 5 , we solve the fourth-order equation for $\rho_{\varepsilon}$, locally in time, with time domain possibly depending on $\varepsilon$. Then, in Section 6 , we prove that, for any $T>0$, the function $\rho_{\varepsilon}$ exists, and is smooth, in the whole of $[0, T]$ provided $\varepsilon$ is small enough. This result is obtained as a consequence of some a priori estimates independent of $\varepsilon$, which we prove in Subsection 6.1. The a priori estimates are also used to prove Main Theorem 1.1 (see Subsection 6.3). Finally, some technical tools are deferred to the appendix.

\section{Notation and function spaces}

In this section we introduce some notation and the function spaces which will be used throughout the paper.

\subsection{Notation}

We define

$$
I=\mathbb{R} \times\left[-\ell_{0} / 2, \ell_{0} / 2\right], \quad I_{-}=(-\infty, 0] \times\left[-\ell_{0} / 2, \ell_{0} / 2\right], \quad I_{+}=[0,+\infty) \times\left[-\ell_{0} / 2, \ell_{0} / 2\right] .
$$

We use the bold notation for elements of both the spaces $C((-\infty, 0]) \times C([0,+\infty))$ and $C\left(I_{-}\right) \times$ $C\left(I_{+}\right)$. Given an element $\mathbf{u}$ of the previous spaces we denote its components by $u_{1}$ and $u_{2}$. We write $D_{x}^{(i)} \mathbf{u}\left(\right.$ resp. $\left.D_{y}^{(i)} \mathbf{u}\right)(i=1,2, \ldots)$ to denote the function whose components are $D_{x}^{(i)} u_{1}$ and $D_{x}^{(i)} u_{2}$ (resp. $D_{y}^{(i)} u_{1}$ and $D_{y}^{(i)} u_{2}$ ).

We extensively use the elements $\mathbf{T}, \mathbf{T}^{\prime}$ (related to the TW), $\mathbf{U}$ and $\mathbf{V}$ of the space $C((-\infty, 0]) \times$ $C([0,+\infty))$, which are defined by

$$
\begin{gathered}
\left\{\begin{array} { l } 
{ T _ { 1 } ( x ) = e ^ { x } , \quad x \leqslant 0 , } \\
{ T _ { 2 } ( x ) = 1 , \quad x \geqslant 0 , }
\end{array} \quad \left\{\begin{array}{l}
T_{1}^{\prime}(x)=e^{x}, \quad x \leqslant 0, \\
T_{2}^{\prime}(x)=0, \quad x \geqslant 0,
\end{array}\right.\right. \\
\left\{\begin{array} { l l } 
{ U _ { 1 } ( x ) = \frac { 1 - x } { 3 } e ^ { x } , } & { x \leqslant 0 , } \\
{ U _ { 2 } ( x ) = \frac { 1 } { 3 } , } & { x \geqslant 0 , }
\end{array} \quad \left\{\begin{array}{ll}
V_{1}(x)=\left(1-\frac{2}{3} x+\frac{x^{2}}{6}\right) e^{x}, & x \leqslant 0, \\
V_{2}(x)=1+\frac{x}{3}, & x \geqslant 0 .
\end{array}\right.\right.
\end{gathered}
$$




\subsection{Function spaces}

Here, we introduce the function spaces we use in this paper.

2.2.1 Spaces of one variable only. Let us fix $\ell_{0}>0$. Given a real- or complex-valued function $f \in L^{2}:=L^{2}\left(-\ell_{0} / 2, \ell_{0} / 2\right)$, we denote by $\widehat{f}(k)$ its $k$-th Fourier coefficient, i.e., we write

$$
f(\eta)=\sum_{k=0}^{+\infty} \widehat{f}(k) w_{k}(\eta), \quad \eta \in\left(-\ell_{0} / 2, \ell_{0} / 2\right),
$$

where $\left\{w_{k}\right\}$ is a complete set of eigenfunctions of the operator

$$
A: D(A)=H^{2} \rightarrow L^{2}, \quad A u=D_{\eta \eta} u, \quad u \in D(A),
$$

with $\ell_{0}$-periodic boundary conditions, corresponding to the sequence $\left\{-\lambda_{k}\right\}$ of nonpositive eigenvalues, which we label as $0=-\lambda_{0}>-\lambda_{1}=-\lambda_{2}>-\lambda_{3}=-\lambda_{4}>\cdots$, for notational convenience.

For integer or arbitrary real $s$ we denote by $H^{s}$ the usual Sobolev spaces of $\ell_{0}$-periodic (generalized) functions, which we conveniently represent as

$$
H^{s}=\left\{w=\sum_{k=0}^{+\infty} a_{k} w_{k}: \sum_{k=0}^{+\infty} \lambda_{k}^{s} a_{k}^{2}<+\infty\right\},
$$

with the usual norm. Next, for any $\beta \geqslant 0$, we denote by $C_{\sharp}^{\beta}$ the space of all functions $f \in C^{\beta}:=$ $C^{\beta}\left(\left[-\ell_{0} / 2, \ell_{0} / 2\right]\right)$ such that $f^{(j)}\left(-\ell_{0} / 2\right)=f^{(j)}\left(\ell_{0} / 2\right)$ for any $j=0, \ldots,[\beta]$. The space $C_{\sharp}^{\beta}$ is endowed with the Euclidean norm of $C^{\beta}\left(\left[-\ell_{0} / 2, \ell_{0} / 2\right]\right)$.

2.2.2 Function spaces of two variables. Given $h, k \in \mathbb{N} \cup\{0\}$, an interval $J \subset \mathbb{R}$ and a (possibly unbounded) closed set $K \subset \mathbb{R}^{d}$ (for some $d \in \mathbb{N}$ ), we denote by $C^{h, k}(J \times K)$ the set of functions $f: J \times K \rightarrow \mathbb{R}$ which are $h$-times continuously differentiable in $J \times K$ with respect to the first variable and $k$-times continuously differentiable in $J \times K$ with respect to the second set of variables. When $J \times K$ is a compact set, we endow the space $C^{h, k}(J \times K)$ with the norm

$$
\|f\|_{C^{h, k}(J \times K)}=\sup _{s \in J}\|f(s, \cdot)\|_{C^{k}(K)}+\sup _{z \in K}\|f(\cdot, z)\|_{C^{h}(J)}
$$

for any $f \in C^{h, k}(J \times K)$. Using 2.4) we can extend the definition of the spaces $C^{h, k}(J \times K)$ to the case when $h, k \notin \mathbb{N}$.

Next, we introduce the space $\mathcal{X}$ defined by

$$
X=\left\{\mathbf{f}=\left(f_{1}, f_{2}\right) \in C\left(I_{-}\right) \times C\left(I_{+}\right): \tilde{f}_{1} \in C_{b}\left(I_{-}\right), \tilde{f}_{2} \in C_{b}\left(I_{+}\right)\right\},
$$

where " $b$ " stands for bounded and the functions $\tilde{f}_{1}$ and $\tilde{f}_{2}$ are defined as follows:

$$
\begin{aligned}
& \tilde{f}_{1}(x, \eta)=e^{-x / 2} f_{1}(x, \eta), \quad x \leqslant 0,|\eta| \leqslant \ell_{0} / 2, \\
& \tilde{f}_{2}(x, \eta)=e^{-x / 2} f_{2}(x, \eta), \quad x \geqslant 0,|\eta| \leqslant \ell_{0} / 2 .
\end{aligned}
$$

In what follows, we will write $\tilde{\mathbf{f}}:=\left(\tilde{f}_{1}, \tilde{f}_{2}\right)$. The space $\mathcal{X}$ is a Banach space when endowed with the norm

$$
\|\mathbf{f}\|_{X}=\left\|\tilde{f}_{1}\right\|_{C_{b}\left(I_{-}\right)}+\left\|\tilde{f}_{2}\right\|_{C_{b}\left(I_{+}\right)}:=\sup _{(x, \eta) \in I_{-}}\left|\tilde{f}_{1}(x, \eta)\right|+\sup _{(x, \eta) \in I_{+}}\left|\tilde{f}_{2}(x, \eta)\right|
$$

for any $\mathbf{f} \in X$. 
2.2.3 Interpolation spaces. Let $X$ be a Banach space and $A: D(A) \subset X \rightarrow X$ be a sectorial operator. For any $\theta \in(0,1)$ we denote by $D_{A}(\theta, \infty)$ the interpolation space between $X$ and $D(A)$, defined as follows:

$$
\begin{aligned}
D_{A}(\theta, \infty) & =\left\{x \in X:[x]_{\theta, \infty}:=\sup _{t \in(0,1]} t^{-\theta}\left\|e^{t A} x-x\right\|<+\infty\right\} \\
& =\left\{x \in X:[[x]]_{\theta, \infty}:=\sup _{t \in(0,1]}\left\|t^{1-\theta} A e^{t A} x\right\|<+\infty\right\},
\end{aligned}
$$

where $\left\{e^{t A}\right\}$ is the analytic semigroup generated by $A . D_{A}(\theta, \infty)$ is a Banach space when endowed with one of the following equivalent norms:

$$
\|x\|_{\theta, \infty, 1}=\|x\|+[x]_{\theta, \infty}, \quad\|x\|_{\theta, \infty, 2}=\|x\|+[[x]]_{\theta, \infty} .
$$

The interpolation space $D_{A}(\theta, \infty)$ can also be defined in a more abstract way as follows:

$$
D_{A}(\theta, \infty):=\left\{x \in X:[[[x]]]_{\theta, \infty}:=\sup _{t \in(0,1]} t^{-\theta} K(t, x)<+\infty\right\},
$$

where

$$
K(t, x)=\inf \left\{\|a\|_{X}+t\|b\|_{D(A)}: x=a+b, a \in X, b \in D(A)\right\},
$$

and $\|\cdot\|_{D(A)}$ denotes the graph-norm. The norm $\|x\|_{\theta, \infty, 3}=\|x\|+[[[x]]]_{\theta, \infty}$ is equivalent to the above defined norms. We refer the reader to [11, Chaps. $1 \& 2]$ and [15] for further details.

\section{Formal Ansatz}

Let us set $\gamma=1-\varepsilon$ in 1.10 . Applying the change of variables defined by (1.12) to problem (1.8)-1.10), the problem for the couple $(v, \psi)$ reads (after simplification by $\varepsilon^{2}$ ) as follows:

$$
v_{x}-\left(v_{x x}+\varepsilon v_{\eta \eta}\right)=\left(\varepsilon \psi_{\tau}+\varepsilon\left(\psi_{\eta}\right)^{2}-\psi_{\eta \eta}\right) \Theta_{x}, \quad x \neq 0,
$$

and at $x=0$ :

$$
\begin{aligned}
& \varepsilon \psi_{\tau}=\left[v_{x}\right]-\varepsilon\left(\psi_{\eta}\right)^{2}, \\
& v_{\mid x=0}=-\psi_{\eta \eta}+\varepsilon \psi_{\eta \eta}+\varepsilon\left(\psi_{\tau}+\frac{1}{2}\left(\psi_{\eta}\right)^{2}\right) .
\end{aligned}
$$

In the spirit of [12, p. 75], we look for formal expansions:

$$
v=v^{0}+\varepsilon v^{1}+\cdots, \quad \psi=\psi^{0}+\varepsilon \psi^{1}+\cdots
$$

of the solution to problem 3.1 - 3.3). Considering the zeroth order part of 3.1 - 3.3) (i.e., the terms with no powers of $\varepsilon$ in front), it is easy to see that the function $v^{0}$ satisfies the system

$$
\begin{aligned}
& v_{x}^{0}-v_{x x}^{0}=-\psi_{\eta \eta}^{0} e^{x} \chi_{(-\infty, 0]}, \\
& {\left[v_{x}^{0}\right]=0,} \\
& v_{\mid x=0}^{0}=-\psi_{\eta \eta}^{0} .
\end{aligned}
$$


It is trivial to solve (3.4) together with, e.g., 3.6): it gives

$$
v^{0}= \begin{cases}-\psi_{\eta \eta}^{0} e^{x}(1-x), & x \leqslant 0 \\ -\psi_{\eta \eta}^{0}, & x>0\end{cases}
$$

We remark that $(3.5)$ is automatically satisfied. Hence, we are unable to "close" the system for $\left(v^{0}, \psi^{0}\right)$ at the zeroth order. This situation is quite common in singular perturbation theory when the zeroth order cannot be fully determined (see, e.g., [7]). In such a case, one needs to go to the first order. Most often, the latter demands a solvability condition, for example based on the Fredholm alternative, which provides the missing relation for the zeroth order. Therefore, repeating computations similar to the previous ones, we get the following equation for $\left(v^{1}, \psi^{1}\right)$ :

$$
v_{x}^{1}-v_{x x}^{1}-v_{\eta \eta}^{0}=\left\{\psi_{\tau}^{0}+\left(\psi_{\eta}^{0}\right)^{2}-\psi_{\eta \eta}^{1}\right\} e^{x} \chi_{(-\infty, 0]} .
$$

At $x=0$ we have the conditions

$$
\begin{aligned}
& {\left[v_{x}^{1}\right]=\psi_{\tau}^{0}+\left(\psi_{\eta}^{0}\right)^{2},} \\
& v_{\mid x=0}^{1}=-\psi_{\eta \eta}^{1}+\psi_{\eta \eta}^{0}+\psi_{\tau}^{0}+\frac{1}{2}\left(\psi_{\eta}^{0}\right)^{2} .
\end{aligned}
$$

Clearly, the solution to 3.7 3.9 is given by

$$
v^{1}= \begin{cases}a e^{x}+2 \psi_{\eta \eta \eta \eta}^{0}-\psi_{\tau}^{0}-\left(\psi_{\eta}^{0}\right)^{2}+\psi_{\eta \eta}^{1} x e^{x}-\frac{1}{2} \psi_{\eta \eta \eta \eta}^{0} x^{2} e^{x}, & x \leqslant 0, \\ a-\psi_{\eta \eta \eta \eta}^{0} x, & x \geqslant 0,\end{cases}
$$

where $a$ is an arbitrary parameter. There are two remaining unknowns at the first order, namely $a$ and $\psi_{\eta \eta}^{1}$, and still two relations at $x=0$. First we use (3.9), which gives

$$
a=v^{1}(0)=-\psi_{\eta \eta}^{1}+\psi_{\eta \eta}^{0}+\psi_{\tau}^{0}+\frac{1}{2}\left(\psi_{\eta}^{0}\right)^{2}
$$

Second, we compute

$$
v_{x}^{1}\left(0^{+}\right)=-\psi_{\eta \eta \eta \eta}^{0} \quad v_{x}^{1}\left(0^{-}\right)=a+2 \psi_{\eta \eta \eta \eta}^{0}-\psi_{\tau}^{0}-\left(\psi_{\eta}^{0}\right)^{2}+\psi_{\eta \eta}^{1} .
$$

Therefore, from 3.8 we get

$$
v_{x}^{1}\left(0^{+}\right)-v_{x}^{1}\left(0^{-}\right)=-a-\left\{3 \psi_{\eta \eta \eta \eta}^{0}-\psi_{\tau}^{0}-\left(\psi_{\eta}^{0}\right)^{2}+\psi_{\eta \eta}^{1}\right\}=\psi_{\tau}^{0}+\left(\psi_{\eta}^{0}\right)^{2} .
$$

Obviously $3.10-3.11$ is a linear system for $\left(a, \psi_{\eta \eta}^{1}\right)$ with solvability condition

$$
\psi_{\eta \eta}^{0}+\psi_{\tau}^{0}+\frac{1}{2}\left(\psi_{\eta}^{0}\right)^{2}+3 \psi_{\eta \eta \eta \eta}^{0}=0
$$

i.e., $\psi^{0}$ satisfies a K-S equation. 


\section{An equivalent problem to 3.1 - 3.3}

The aim of this section is to transform problem $3.1-3.3$ into an equivalent one. More precisely, we are going to decouple the problem, getting a self-consistent equation for the front $\psi$ and an equation for the other unknown (say $\mathbf{z}$ ) which can be immediately solved once $\psi$ is known.

In deriving the equivalent problem, we assume that the solution $(v, \psi)$ to problem $3.1-3.3$ in the time domain $[0, T]$ belongs to the space $\nu_{T} \times y_{T}$, defined as follows:

DEFINITION 4.1 For any $T>0$, we denote by $\mathcal{V}_{T}$ the space of all functions $v:[0, T] \times \mathbb{R} \times$ $\left[-\ell_{0} / 2, \ell_{0} / 2\right] \rightarrow \mathbb{R}$ such that

(i) $v$ is twice continuously differentiable with respect to the spatial variables in $[0, T] \times I_{-}$and in $[0, T] \times I_{+}$

(ii) the functions $(\tau, x, \eta) \mapsto e^{-x / 2} D_{x}^{(i)} v(\tau, x, \eta)$ and $(\tau, x, \eta) \mapsto e^{-x / 2} D_{\eta}^{(i)} v(\tau, x, \eta)$ are bounded in $[0, T] \times I_{-}$and in $[0, T] \times I_{+}$for any $i=0,1,2$.

Further, for any $\alpha \in(0,1 / 2)$, we denote by $y_{T}$ the space of all functions $\zeta \in C^{1,4}([0, T] \times$ $\left.\left[-\ell_{0} / 2, \ell_{0} / 2\right]\right)$ such that $\zeta_{\tau} \in C^{0,2+\alpha}\left([0, T] \times\left[-\ell_{0} / 2, \ell_{0} / 2\right]\right)$ and $D_{\eta}^{(j)} \zeta\left(\cdot,-\ell_{0} / 2\right)=$ $D_{\eta}^{(j)} \zeta\left(\cdot, \ell_{0} / 2\right)$ for $j=0,1,2,3$.

REMARK 4.2 Note that, for any $\zeta \in y_{T}$, the function $\zeta_{\eta \eta}$ is continuously differentiable in $[0, T] \times$ $\left[-\ell_{0} / 2, \ell_{0} / 2\right]$ with respect to $\tau$, and $\zeta_{\tau \eta \eta} \equiv \zeta_{\eta \eta \tau}$. Indeed,

$$
\zeta(\tau, \eta)=\zeta(0, \eta)+\int_{0}^{\tau} \zeta_{\tau}(s, \eta) \mathrm{d} s, \quad \tau \in[0, T], \eta \in[-\ell / 2, \ell / 2] .
$$

Since $\zeta_{\tau} \in C^{0,2}([0, T] \times[-\ell / 2, \ell / 2])$ we can differentiate under the integral sign to get

$$
\zeta_{\eta \eta}(\tau, \eta)=\zeta_{\eta \eta}(0, \eta)+\int_{0}^{\tau} \zeta_{\eta \eta \tau}(s, \eta) \mathrm{d} s, \quad \tau \in[0, T], \eta \in[-\ell / 2, \ell / 2] .
$$

This formula clearly shows that $\zeta_{\eta \eta}$ is continuously differentiable with respect to $\tau$ in $[0, T] \times$ $[-\ell / 2, \ell / 2]$ and $\zeta_{\tau \eta \eta} \equiv \zeta_{\eta \eta \tau}$. Hence, in what follows, we always write $\zeta_{\tau \eta \eta}$ instead of $\zeta_{\eta \eta \tau}$.

\subsection{Derivation of a self-consistent equation for the front}

In this subsection we derive a self-consistent equation for the front. Since its derivation is rather long, we split the proof into several steps.

4.1.1 Elimination of $\psi_{\tau}$. First we eliminate $\psi_{\tau}$ from 3.1) thanks to 3.3, getting the equation

$$
v_{x}-v_{x x}-\varepsilon v_{\eta \eta}-v(\cdot, 0, \cdot) \Theta_{x}=\left(\frac{1}{2} \varepsilon\left(\psi_{\eta}\right)^{2}-\varepsilon \psi_{\eta \eta}\right) \Theta_{x}, \quad x \neq 0
$$

Let us set $\mathbf{v}(\tau, x, \eta):=\left(v_{1}(\tau, x, \eta), v_{2}(\tau, x, \eta)\right)$, where $v_{1}(\cdot, x, \cdot)=v(\cdot, x, \cdot)$ for any $x \leqslant 0$ and $v_{2}(\cdot, x, \cdot)=v(\cdot, x, \cdot)$ for any $x \geqslant 0$, and

$$
\mathbf{F}_{0}=\left(\psi_{\eta \eta}-\frac{1}{2}\left(\psi_{\eta}\right)^{2}\right) \mathbf{T}^{\prime}, \quad g=\psi_{\tau}+\left(\psi_{\eta}\right)^{2},
$$


where $\mathbf{T}^{\prime}$ is given by $(2.1)$. Taking $(3.3)$ and $(4.1)$ into account, we can easily show that $\mathbf{v}$ solves the problem

$$
\left\{\begin{array}{l}
\mathcal{L} \mathbf{v}=\varepsilon \mathbf{F}_{0}-\varepsilon \mathbf{v}_{\eta \eta} \\
v_{2}(\cdot, 0, \cdot)-v_{1}(\cdot, 0, \cdot)=0 \\
D_{x} v_{2}(\cdot, 0, \cdot)-D_{x} v_{1}(\cdot, 0, \cdot)=\varepsilon g
\end{array}\right.
$$

where

$$
(\mathcal{L} \mathbf{v})(\cdot, x, \eta)= \begin{cases}D_{x x} v_{1}(\cdot, x, \eta)-D_{x} v_{1}(\cdot, x, \eta)+e^{x} v_{1}(\cdot, 0, \eta), & x \leqslant 0,|\eta| \leqslant \ell_{0} / 2, \\ D_{x x} v_{2}(\cdot, x, \eta)-D_{x} v_{2}(\cdot, x, \eta), & x \geqslant 0,|\eta| \leqslant \ell_{0} / 2 .\end{cases}
$$

4.1.2 Lifting up the boundary conditions. We introduce the new unknown $\mathbf{w}=\mathbf{v}-\varepsilon \mathcal{N}(g)$, where $\mathcal{N}(g)=g(\mathbf{V}-\mathbf{T})$, and $\mathbf{T}$ and $\mathbf{V}$ are defined in 2.1] and 2.2). It is easy to see that $\mathbf{w}$ solves the problem

$$
\left\{\begin{array}{l}
\mathcal{L} \mathbf{w}=\varepsilon \mathbf{F}_{0}-\varepsilon \mathbf{w}_{\eta \eta}-\varepsilon^{2} g_{\eta \eta} \mathcal{N}(1)-\varepsilon g \mathcal{L} \mathcal{N}(1) \\
w_{2}(\cdot, 0, \cdot)-w_{1}(\cdot, 0, \cdot)=0 \\
D_{x} w_{2}(\cdot, 0, \cdot)-D_{x} w_{1}(\cdot, 0, \cdot)=0
\end{array}\right.
$$

Since $\mathbf{v} \in \mathcal{V}_{T}, \mathbf{v}(\tau, \cdot), \mathcal{L} \mathbf{v}(\tau, \cdot) \in X$ (see 2.5 for the definition of the space $X$ ) for any $\tau \in[0, T]$, a straightforward computation shows that $\mathbf{w}(\tau, \cdot)$ belongs to $X$ for any $\tau \in[0, T]$, and hence to the set

$$
\left\{\mathbf{h} \in C^{2,0}\left(I_{-}\right) \times C^{2,0}\left(I_{+}\right): \mathbf{h}, \mathcal{L} \mathbf{h} \in X, D_{x}^{(j)} h_{1}(0, \cdot)=D_{x}^{(j)} h_{2}(0, \cdot), j=0,1\right\},
$$

which is the domain of the realization $L$ of the operator $\mathcal{L}$ in $X$ (see Section A.1).

4.1.3 A Lyapunov-Schmidt method. From the results in the previous subsection, we know that $\mathbf{w}(\tau, \cdot) \in D(L)$ for any $\tau \in[0, T]$, and it solves the equation

$$
L \mathbf{w}=\varepsilon \mathbf{F}_{0}-\varepsilon \mathbf{w}_{\eta \eta}-\varepsilon^{2} g_{\eta \eta} \mathcal{N}(1)-\varepsilon g \mathcal{L} \mathcal{N}(1)
$$

We are going to project (4.4) along a suitable subspace of $\mathcal{X}$, to derive a self-consistent equation for the front $\psi$.

According to Theorem A.1 the equation $L \mathbf{z}=\mathbf{f} \in X$ has a solution if and only if $\mathcal{P}(\mathbf{f})=0$, where

$$
\mathcal{P}(\mathbf{f})=\left(\int_{-\infty}^{0} f_{1}(x, \cdot) \mathrm{d} x+\int_{0}^{+\infty} e^{-x} f_{2}(x, \cdot) \mathrm{d} x\right) \mathbf{U}=Q(\mathbf{f}) \mathbf{U}, \quad \mathbf{f} \in X .
$$

Since $\mathbf{w}$ is any of such solutions, it follows that

$$
\mathcal{P}\left(\varepsilon \mathbf{F}_{0}-\varepsilon \mathbf{w}_{\eta \eta}-\varepsilon g \mathcal{L} \mathcal{N}(1)-\varepsilon^{2} g_{\eta \eta} \mathcal{N}(1)\right)=0,
$$

or equivalently, after division by $\varepsilon>0$,

$$
0=Q\left(\mathbf{F}_{0}-\mathbf{w}_{\eta \eta}-g \mathcal{L} \mathcal{N}(1)-\varepsilon g_{\eta \eta} \mathcal{N}(1)\right) .
$$

Observing that

$$
\begin{aligned}
& Q\left(\mathbf{F}_{0}\right)=\psi_{\eta \eta}-\frac{1}{2}\left(\psi_{\eta}\right)^{2}, \\
& Q\left(g_{\eta \eta} \mathcal{N}(1)\right)=\frac{4}{3} g_{\eta \eta}=\frac{4}{3}\left(\psi_{\tau \eta \eta}+\left(\left(\psi_{\eta}\right)^{2}\right)_{\eta \eta}\right), \\
& Q(g \mathcal{L} \mathcal{N}(1))=-g=-\psi_{\tau}-\left(\psi_{\eta}\right)^{2},
\end{aligned}
$$


we can rewrite (4.5) as follows:

$$
\psi_{\tau}-\frac{4}{3} \varepsilon \psi_{\tau \eta \eta}+\frac{1}{2}\left(\psi_{\eta}\right)^{2}+\psi_{\eta \eta}-\frac{4}{3} \varepsilon\left(\left(\psi_{\eta}\right)^{2}\right)_{\eta \eta}=Q\left(\mathbf{w}_{\eta \eta}\right)
$$

To get a self-contained equation for the front $\psi$, we have to give a representation of $Q\left(\mathbf{w}_{\eta \eta}\right)$ on the right-hand side of 4.7). For this purpose, in the spirit of the Lyapunov-Schmidt method, we split $\mathbf{w}(\tau, \cdot)(\tau \in[0, T])$ along $\mathcal{P}(X)$ and $(I-\mathcal{P})(X)$. Writing

$$
\mathbf{w}(\tau, x, \eta)=a(\tau, \eta) \mathbf{U}(x)+\varepsilon \mathbf{z}(\tau, x, \eta),
$$

and observing that our assumptions on $v$ guarantee that $\mathbf{z}_{\eta \eta}$ belongs to $(I-\mathcal{P})(\mathcal{X})$ (indeed, $\mathcal{P} \mathbf{z}=0$ and $\mathcal{P}$ commutes with $D_{\eta}$ ), we get

$$
Q\left(\mathbf{w}_{\eta \eta}\right)=Q\left(a_{\eta \eta} \mathbf{U}+\varepsilon \mathbf{z}_{\eta \eta}\right)=a_{\eta \eta} .
$$

Let us compute formally the function $a$ and its second spatial derivative. We use the relation in (3.3) to obtain

$$
\frac{1}{3} a+\varepsilon z_{1}(\cdot, 0, \cdot)=(\varepsilon-1) \psi_{\eta \eta}+\varepsilon \psi_{\tau}+\frac{1}{2} \varepsilon\left(\psi_{\eta}\right)^{2} .
$$

Thus,

$$
a_{\eta \eta}=-3 \varepsilon D_{\eta \eta} z_{1}(\cdot, 0, \cdot)+3(\varepsilon-1) \psi_{\eta \eta \eta \eta}+3 \varepsilon \psi_{\tau \eta \eta}+\frac{3}{2} \varepsilon\left(\left(\psi_{\eta}\right)^{2}\right)_{\eta \eta}
$$

From (4.8) and 4.9) it follows that

$$
Q\left(\mathbf{w}_{\eta \eta}\right)=-3 \varepsilon D_{\eta \eta} z_{1}(\cdot, 0, \cdot)+3(\varepsilon-1) \psi_{\eta \eta \eta \eta}+3 \varepsilon \psi_{\tau \eta \eta}+\frac{3}{2} \varepsilon\left(\left(\psi_{\eta}\right)^{2}\right)_{\eta \eta} .
$$

Inserting this into 4.7 we get the following equation for $\psi$ :

$$
\psi_{\tau}-\frac{13}{3} \varepsilon \psi_{\tau \eta \eta}+3(1-\varepsilon) \psi_{\eta \eta \eta \eta}+\psi_{\eta \eta}+\frac{1}{2}\left(\psi_{\eta}\right)^{2}+3 \varepsilon D_{\eta \eta} z_{1}(\cdot, 0, \cdot)=\frac{17}{6} \varepsilon\left(\left(\psi_{\eta}\right)^{2}\right)_{\eta \eta} .
$$

We already see that 4.10 reduces to K-S if $\varepsilon=0$. However, we still have $z_{1}$ on the left-hand side of 4.10. In the next subsection, we write it in terms of $\psi$.

4.1.4 The equation for $\mathbf{z}$. To write $D_{\eta \eta} z_{1}(\cdot, 0, \cdot)$ in terms of $\psi$, we determine the equation satisfied by $\mathbf{z}$. Projecting equation 4.4 on $(I-\mathcal{P})(\mathcal{X})$, we see that $\mathbf{z}(\tau, \cdot)=(I-\mathcal{P})(\mathbf{z}(\tau, \cdot)) \in D(L)$ $(\tau \in[0, T])$ solves the equation

$$
L \mathbf{z}=(I-\mathcal{P})\left(\mathbf{F}_{0}\right)-g(I-\mathcal{P})(\mathcal{L} \mathcal{N}(1))-\varepsilon g_{\eta \eta}(I-\mathcal{P})(\mathcal{N}(1))-\varepsilon \mathbf{z}_{\eta \eta} .
$$

Taking 4.6a-4.6c into account, we can rewrite 4.11) as

$$
L \mathbf{z}+\varepsilon \mathbf{z}_{\eta \eta}=\left(\psi_{\eta \eta}-\frac{1}{2}\left(\psi_{\eta}\right)^{2}\right)\left(\mathbf{T}^{\prime}-\mathbf{U}\right)-\varepsilon\left(\psi_{\tau \eta \eta}+\left(\left(\psi_{\eta}\right)^{2}\right)_{\eta \eta}\right)\left(\mathbf{V}-\mathbf{T}-\frac{4}{3} \mathbf{U}\right)
$$

We now observe that the operator $L+\varepsilon A:=L+\varepsilon D_{\eta \eta}$ with domain

$$
D(L+\varepsilon A)=\left\{\mathbf{u} \in D(L): \mathbf{u}_{\eta \eta} \in \mathcal{X}, D_{\eta}^{(j)} u_{i}\left(\cdot,-\ell_{0} / 2\right)=D_{\eta}^{(j)} u_{i}\left(\cdot, \ell_{0} / 2\right), i=1,2, j=0,1\right\}
$$


is closable and its closure, denoted by $L_{\varepsilon}$, is sectorial and 0 is in the resolvent set of the restriction of $L_{\varepsilon}$ to $(I-\mathcal{P})(X)$ (see Theorem A.2). Hence, we can invert (4.12) using $R\left(0, L_{\varepsilon}\right)=\left(-L_{\varepsilon}\right)^{-1}$, collecting linear and nonlinear terms in $\psi$ :

$$
\begin{aligned}
\mathbf{z}= & R\left(0, L_{\varepsilon}\right)\left(-\psi_{\eta \eta}\left(\mathbf{T}^{\prime}-\mathbf{U}\right)+\varepsilon \psi_{\tau \eta \eta}\left(\mathbf{V}-\mathbf{T}-\frac{4}{3} \mathbf{U}\right)\right) \\
& +R\left(0, L_{\varepsilon}\right)\left(\frac{1}{2}\left(\psi_{\eta}\right)^{2}\left(\mathbf{T}^{\prime}-\mathbf{U}\right)+\varepsilon\left(\left(\psi_{\eta}\right)^{2}\right)_{\eta \eta}\left(\mathbf{V}-\mathbf{T}-\frac{4}{3} \mathbf{U}\right)\right) .
\end{aligned}
$$

4.1.5 The fourth-order equation for the front. Using (4.14), we can compute $z_{1}(\cdot, 0, \cdot)$ getting

$$
\begin{aligned}
z_{1}(\cdot, 0, \cdot)= & -\left(R\left(0, L_{\varepsilon}\right)\left[\psi_{\eta \eta}\left(\mathbf{T}^{\prime}-\mathbf{U}\right)\right]\right)_{1}(\cdot, 0, \cdot)+\varepsilon\left(R\left(0, L_{\varepsilon}\right)\left[\psi_{\tau \eta \eta}\left(\mathbf{V}-\mathbf{T}-\frac{4}{3} \mathbf{U}\right)\right]\right)_{1}(\cdot, 0, \cdot) \\
& +\left\{R\left(0, L_{\varepsilon}\right)\left(\frac{1}{2}\left(\psi_{\eta}\right)^{2}\left(\mathbf{T}^{\prime}-\mathbf{U}\right)+\varepsilon\left(\left(\psi_{\eta}\right)^{2}\right)_{\eta \eta}\left(\mathbf{V}-\mathbf{T}-\frac{4}{3} \mathbf{U}\right)\right)\right\}_{1}(\cdot, 0, \cdot) .
\end{aligned}
$$

Since $z_{1}$ is as smooth as $v_{1}$ is, we can differentiate the previous formula twice with respect to $\eta$ obtaining

$$
\begin{aligned}
D_{\eta \eta} z_{1}(\cdot, 0, \cdot)= & -\left(D_{\eta \eta} R\left(0, L_{\varepsilon}\right)\left[\psi_{\eta \eta}\left(\mathbf{T}^{\prime}-\mathbf{U}\right)\right]\right)_{1}(\cdot, 0, \cdot) \\
& +\varepsilon\left(D_{\eta \eta} R\left(0, L_{\varepsilon}\right)\left[\psi_{\tau \eta \eta}\left(\mathbf{V}-\mathbf{T}-\frac{4}{3} \mathbf{U}\right)\right]\right)_{1}(\cdot, 0, \cdot) \\
& +\frac{1}{2}\left\{D_{\eta \eta} R\left(0, L_{\varepsilon}\right)\left(\left(\psi_{\eta}\right)^{2}\left(\mathbf{T}^{\prime}-\mathbf{U}\right)\right)\right\}_{1}(\cdot, 0, \cdot) \\
& +\varepsilon\left\{D_{\eta \eta} R\left(0, L_{\varepsilon}\right)\left(\left(\left(\psi_{\eta}\right)^{2}\right)_{\eta \eta}\left(\mathbf{V}-\mathbf{T}-\frac{4}{3} \mathbf{U}\right)\right)\right\}_{1}(\cdot, 0, \cdot) .
\end{aligned}
$$

Estimate $\mathrm{A} 2$ ) and our assumptions on $\psi$ (which guarantee that $\psi_{\eta \eta} \in C^{1}\left([0, T] ; C^{\alpha}\right)$, see Remark 4.2 show that $\left(D_{\eta \eta} R\left(0, L_{\varepsilon}\right)\left[\psi_{\eta \eta}\left(\mathbf{V}-\mathbf{T}-\frac{4}{3} \mathbf{U}\right)\right]\right)_{1}(\cdot, 0, \cdot)$ belongs to $C^{1,0}\left([0, T] \times\left[-\ell_{0} / 2, \ell_{0} / 2\right]\right)$ and its derivative is $\left(D_{\eta \eta} R\left(0, L_{\varepsilon}\right)\left[\psi_{\tau \eta \eta}\left(\mathbf{V}-\mathbf{T}-\frac{4}{3} \mathbf{U}\right)\right]\right)_{1}(\cdot, 0, \cdot)$. Hence, inserting (4.15) into (4.10), we conclude that the function $\psi$ solves the fourth-order equation

$$
\frac{\partial}{\partial \tau} \mathcal{B}_{\varepsilon} \psi=\mathcal{S}_{\varepsilon} \psi+\mathcal{F}_{\varepsilon}\left(\left(\psi_{\eta}\right)^{2}\right)
$$

where

$$
\begin{aligned}
\mathcal{B}_{\varepsilon} \psi= & \psi-\frac{13 \varepsilon}{3} \psi_{\eta \eta}+3 \varepsilon^{2}\left(D_{\eta \eta} R\left(0, L_{\varepsilon}\right)\left[\psi_{\eta \eta}\left(\mathbf{V}-\mathbf{T}-\frac{4}{3} \mathbf{U}\right)\right]\right)_{1}(\cdot, 0, \cdot), \\
\mathcal{S}_{\varepsilon} \psi= & -3(1-\varepsilon) \psi_{\eta \eta \eta \eta}-\psi_{\eta \eta}+3 \varepsilon\left(D_{\eta \eta} R\left(0, L_{\varepsilon}\right)\left[\psi_{\eta \eta}\left(\mathbf{T}^{\prime}-\mathbf{U}\right)\right]\right)_{1}(\cdot, 0, \cdot), \\
\mathcal{F}_{\varepsilon}(\psi)= & -3 \varepsilon D_{\eta \eta}\left\{R\left(0, L_{\varepsilon}\right)\left(\frac{1}{2} \psi\left(\mathbf{T}^{\prime}-\mathbf{U}\right)+\varepsilon \psi_{\eta \eta}\left(\mathbf{V}-\mathbf{T}-\frac{4}{3} \mathbf{U}\right)\right)\right\}_{1}(\cdot, 0, \cdot) \\
& +\frac{17 \varepsilon}{6} \psi_{\eta \eta}-\frac{1}{2} \psi
\end{aligned}
$$

Clearly, 4.16 reduces to K-S when we set $\varepsilon=0$. 


\subsection{Equivalence between problem (3.1)-3.3 and equation 4.16}

The following theorem states the equivalence of problem (3.1)-3.3 and equation 4.16 .

TheOREM 4.3 Fix $\varepsilon, T>0$ and $\alpha \in(0,1 / 2)$. Further, let $(v, \psi) \in \nu_{T} \times y_{T}$ be a solution to problem (3.1)-3.3) (see Definition 4.1). Then the function $\psi$ solves (4.16). Conversely, if $\psi \in y_{T}$ is a solution to (4.16), then there exists a function $v \in \mathcal{V}_{T}$ such that the pair $(v, \psi)$ solves the Cauchy problem $3.1-3.3$.

Proof. In view of the arguments in Subsection 4.1, we just need to show that to any solution $\psi \in y_{T}$ to equation 4.16 there corresponds a unique function $v \in \mathcal{V}_{T}$ such that the pair $(v, \psi)$ solves problem (3.1)-(3.3). For this purpose, let $\mathbf{z}$ be defined by (4.14). By assumptions, $\psi_{\eta \eta},\left(\psi_{\eta}\right)^{2}$, $\left(\left(\psi_{\eta}\right)^{2}\right)_{\eta \eta}$ and $\psi_{\tau \eta \eta}$ are bounded in $[0, T]$ with values in $C_{\sharp}^{\alpha}$. Moreover, the functions $\mathbf{T}^{\prime}-\mathbf{U}$ and $\mathbf{V}-\mathbf{T}-\frac{4}{3} \mathbf{U}$ are in $(I-\mathcal{P})(\mathcal{X})$. Hence, we can apply Theorem A.2 (iii) to conclude that $\mathbf{z}(\tau, \cdot)$ is in $D(L+\varepsilon A)($ see 4.13$)$ ) for any $\tau \in[0, T]$.

Clearly, the components $z_{1}$ and $z_{2}$ of $\mathbf{z}$ are continuous in $[0, T] \times I_{-}$and $[0, T] \times I_{+}$, respectively. Let us show that also their spatial derivatives (up to the second order) are continuous in $[0, T] \times I_{-}$ and $[0, T] \times I_{+}$, respectively. This follows from the estimate $\mathrm{A} 2$ provided one shows that the functions $\psi_{\eta \eta},\left(\left(\psi_{\eta}\right)^{2}\right)_{\eta \eta}$ and $\psi_{\tau \eta \eta}$ belong to $C\left([0, T] ; C_{\sharp}^{\theta}\right)$ for some $\theta \in(0, \alpha)$. This property can be proved using an interpolation argument. Indeed, it is well-known that, for any $\theta \in(0, \alpha)$, there exists a positive constant $K$ such that

$$
\|\psi\|_{C^{\theta}} \leqslant K\|\psi\|_{C^{0}}^{1-\theta / \alpha}\|\psi\|_{C^{\alpha}}^{\theta / \alpha}
$$

for any $\psi \in C^{\alpha}$ (see, e.g., [15]). Applying this estimate to the function $\psi_{\eta \eta}\left(\tau_{2}, \cdot\right)-\psi_{\eta \eta}\left(\tau_{1}, \cdot\right)$, with $\tau_{1}, \tau_{2} \in[0, T]$, shows that $\psi_{\eta \eta}$ is continuous in $[0, T]$ with values in $C^{\theta}$ (and, hence, in $C_{\sharp}^{\theta}$ ) for any $\theta \in(0, \alpha)$. The same argument shows that the functions $\left(\psi_{\eta}\right)^{2},\left(\left(\psi_{\eta}\right)^{2}\right)_{\eta \eta}$ and $\psi_{\tau \eta \eta}$ are in $C\left([0, T] ; C_{\sharp}^{\theta}\right)$ as well. Finally, since $\mathbf{z}(\tau, \cdot) \in D(L+\varepsilon A)$ for any $\tau \in[0, T]$, the functions $(\tau, x, \eta) \mapsto e^{-x / 2} D_{x}^{(i)} z_{1}(\tau, x, \eta)$ and $(\tau, x, \eta) \mapsto e^{-x / 2} D_{\eta}^{(i)} z_{2}(\tau, x, \eta)$ are bounded in $[0, T] \times I_{-}$ and $[0, T] \times I_{+}$, respectively, for any $i=0,1,2$.

$\mathbf{z}$ will represent the component along $(I-\mathcal{P})(\mathcal{X})$ of $\mathbf{v}-\varepsilon \mathcal{N}\left(\psi_{\tau}+\left(\psi_{\eta}\right)^{2}\right)$, where $v_{1}(\cdot, x, \cdot)=$ $v(\cdot, x, \cdot)$ for any $x \leqslant 0$ and $v_{2}(\cdot, x, \cdot)=v(\cdot, x, \cdot)$ for any $x \geqslant 0$, and $v$ is the solution to problem 3.1 -3.3 we are looking for. The computations in Subsection 4.1 suggest setting $\mathbf{v}:=\mathbf{w}+\varepsilon \mathcal{N}\left(\psi_{\tau}+\left(\psi_{\eta}\right)^{2}\right):=a \mathbf{U}+\varepsilon \mathbf{z}+\varepsilon \mathcal{N}\left(\psi_{\tau}+\left(\psi_{\eta}\right)^{2}\right)$, where

$$
a=-3 \varepsilon z_{1}(\cdot, 0, \cdot)+3(\varepsilon-1) \psi_{\eta \eta}+3 \varepsilon \psi_{\tau}+\frac{3}{2} \varepsilon\left(\psi_{\eta}\right)^{2} .
$$

Using formulae 4.6a-4.6c) and 4.15 we can show that

$$
\mathcal{P}\left(\varepsilon \mathbf{F}_{0}-\varepsilon \mathbf{w}_{\eta \eta}-\varepsilon \mathcal{L} \mathcal{N}\left(\psi_{\tau}+\left(\psi_{\eta}\right)^{2}\right)-\varepsilon^{2} \mathcal{N}\left(\left(\psi_{\tau}+\left(\psi_{\eta}\right)^{2}\right)_{\eta \eta}\right)\right)=0 .
$$

Hence, $\mathbf{v}$ solves the equation

$$
\begin{aligned}
\mathcal{L} \mathbf{v} & =L(a \mathbf{U})+\varepsilon L \mathbf{z}+\varepsilon \mathcal{L} \mathcal{N}\left(\psi_{\tau}+\left(\psi_{\eta}\right)^{2}\right) \\
& =(I-\mathcal{P})\left\{\varepsilon \mathbf{F}_{0}-\varepsilon \mathbf{w}_{\eta \eta}-\varepsilon \mathcal{L} \mathcal{N}\left(\psi_{\tau}+\left(\psi_{\eta}\right)^{2}\right)-\varepsilon^{2} \mathcal{N}\left(\left(\psi_{\tau}+\left(\psi_{\eta}\right)^{2}\right)_{\eta \eta}\right)\right\}+\varepsilon \mathcal{L}\left(\mathcal{N}\left(\psi_{\tau}+\left(\psi_{\eta}\right)^{2}\right)\right) \\
& =\varepsilon \mathbf{F}_{0}-\varepsilon \mathbf{v}_{\eta \eta} .
\end{aligned}
$$


Moreover, it is easy to check that $\mathbf{v}$ also satisfies the boundary conditions of the Cauchy problem (4.2).

Clearly, the function $v$ defined above belongs to $\mathcal{V}_{T}$ and the pair $(v, \psi)$ solves the differential equation (3.1). Using the second boundary condition in (4.2), it follows immediately that $(v, \psi)$ satisfies condition (3.2). Finally, to check condition (3.3) it suffices to use (4.18), recalling that $\mathcal{N}\left(\psi_{\tau}+\left(\psi_{\eta}\right)^{3}\right)$ vanishes when $\eta=0$. This completes the proof.

\subsection{The equation for the remainder}

In view of Theorem 4.3 in the rest of the paper we deal only with equation (4.16) with periodic boundary conditions. To begin, we recall the following result about K-S:

TheOREM 4.4 Let $\Phi_{0} \in C_{\sharp}^{6+\alpha}$ for some $\alpha \in(0,1 / 2)$. Then the Cauchy problem

$$
\begin{cases}\Phi_{\tau}(\tau, \eta)=-3 \Phi_{\eta \eta \eta \eta}(\tau, \eta)-\Phi_{\eta \eta}(\tau, \eta)-\frac{1}{2}\left(\Phi_{\eta}(\tau, \eta)\right)^{2}, & \tau \geqslant 0,|\eta| \leqslant \ell_{0} / 2, \\ D_{\eta}^{k} \Phi\left(\tau,-\ell_{0} / 2\right)=D_{\eta}^{k} \Phi\left(\tau, \ell_{0} / 2\right), & \tau \geqslant 0, k=0,1,2,3, \\ \Phi(0, \eta)=\Phi_{0}(\eta), & |\eta| \leqslant \ell_{0} / 2,\end{cases}
$$

admits a unique solution $\Phi \in C^{1,4}\left([0,+\infty) \times\left[-\ell_{0} / 2, \ell_{0} / 2\right]\right)$. In fact, $\Phi \in y_{T}$ for any $T>0$.

Most of the literature is about the differentiated version of K-S. For this reason and the reader's convenience, we provide a full proof of Theorem 4.4 in the appendix.

According to the Ansatz, we split

$$
\psi=\Phi+\varepsilon \rho_{\varepsilon},
$$

which defines the remainder $\rho_{\varepsilon}$. To avoid cumbersome notation, we simply write $\rho$ for $\rho_{\varepsilon}$. From Theorem 4.4 we know that $\rho \in y_{T}$ and it solves the equation

$$
\frac{\partial}{\partial \tau} \mathcal{B}_{\varepsilon}(\rho)=\mathcal{S}_{\varepsilon}(\rho)-\Phi_{\eta} \rho_{\eta}-\frac{\varepsilon}{2}\left(\rho_{\eta}\right)^{2}+\mathcal{G}_{\varepsilon}\left(\left(\Phi_{\eta}+\varepsilon \rho_{\eta}\right)^{2}\right)+\mathcal{H}_{\varepsilon}(\Phi)
$$

where

$$
\begin{aligned}
\mathcal{G}_{\varepsilon}(\xi)= & \frac{17}{6} \xi_{\eta \eta}-3\left\{D_{\eta \eta} R\left(0, L_{\varepsilon}\right)\left(\frac{1}{2} \xi\left(\mathbf{T}^{\prime}-\mathbf{U}\right)+\varepsilon \xi_{\eta \eta}\left(\mathbf{V}-\mathbf{T}-\frac{4}{3} \mathbf{U}\right)\right)\right\}_{1}(\cdot, 0, \cdot), \\
\mathcal{H}_{\varepsilon}(\Phi)= & 3 \Phi_{\eta \eta \eta \eta}+3\left(D_{\eta \eta} R\left(0, L_{\varepsilon}\right)\left[\Phi_{\eta \eta}\left(\mathbf{T}^{\prime}-\mathbf{U}\right)\right]\right)_{1}(\cdot, 0, \cdot)+\frac{13}{3} \Phi_{\tau \eta \eta} \\
& -3 \varepsilon\left(D_{\eta \eta} R\left(0, L_{\varepsilon}\right)\left[\Phi_{\tau \eta \eta}\left(\mathbf{V}-\mathbf{T}-\frac{4}{3} \mathbf{U}\right)\right]\right)_{1}(\cdot, 0, \cdot) .
\end{aligned}
$$

Equation 4.20 on $\left[-\ell_{0} / 2, \ell_{0} / 2\right]$ is supplemented by periodic boundary conditions and by an initial condition $\rho_{0}$ at $\tau=0$. For simplicity, to avoid lengthly computations, we take hereafter $\rho_{0}=0$, that is, $\psi(0, \cdot)=\Phi(0, \cdot)=\Phi_{0}$. In other words, the front $\psi$ and the solution of K-S start from the same configuration, which is physically reasonable. More general compatible initial data can be considered as in [3, 1]. 


\section{Local in time solvability of equation (4.20)}

As has been remarked in the introduction, except for small $\ell_{0}$, where the TW is stable, global existence of $\rho$ is not guaranteed.

In this section, we prove the following local in time existence and uniqueness result.

THEOREM 5.1 For any $\varepsilon \in(0,1 / 2]$ there exist $T_{\varepsilon}>0$ and a unique solution $\rho$ to equation 4.20) which belongs to $y_{T_{\varepsilon}}$ and vanishes at $\tau=0$.

The proof is rather long and needs many preliminary results. For this reason, we split it into several steps. Before entering into the details, we sketch here the strategy of the proof.

As a first step, for any fixed $\varepsilon>0$, we transform (4.20) into a semilinear equation associated with a sectorial operator. Employing classical tools from the theory of analytic semigroups we prove that such a semilinear equation admits a unique solution $\rho=\rho_{\varepsilon}$ defined in some time domain $\left[0, T_{\varepsilon}\right]$, which vanishes at $\tau=0$. Using some bootstrap arguments, we then regularize $\rho$, showing that it actually belongs to $y_{T_{\varepsilon}}$. These regularity properties of $\rho$ allow us to show that it is in fact a solution to 4.20].

\subsection{The semilinear equation}

In this subsection, we show that we can transform equation (4.20) into a semilinear equation associated with a second order elliptic operator. We obtain it by inverting the operator $\mathcal{B}_{\varepsilon}$ in $4.17 \mathrm{a}$, i.e., the operator defined by

$$
\mathcal{B}_{\varepsilon} \psi=\psi-\frac{13 \varepsilon}{3} \psi_{\eta \eta}+3 \varepsilon^{2}\left(D_{\eta \eta} R\left(0, L_{\varepsilon}\right)\left[\psi_{\eta \eta}\left(\mathbf{V}-\mathbf{T}-\frac{4}{3} \mathbf{U}\right)\right]\right)_{1}(\cdot, 0, \cdot) .
$$

By Theorem A.2 and the results in the proof of Theorem 4.3 , we know that the operator $\mathcal{B}_{\varepsilon}$ is welldefined in $C_{\sharp}^{2+\theta}$ for any $\theta \in(0,1)$. We will show that $\mathcal{B}_{\varepsilon}$ can be extended to the whole of $C_{\sharp}^{2}$ as an operator which is invertible. For this purpose, we compute the symbol of the operator $\mathcal{B}_{\varepsilon}$.

Throughout the section, given a function $f: J \times\left[-\ell_{0} / 2, \ell_{0} / 2\right] \rightarrow \mathbb{R}$, where $J \subset \mathbb{R}$ is an interval, we denote by $\widehat{f}(x, k)$ the $k$-th Fourier coefficient of the function $f(x, \cdot)$. Moreover, we set

$$
X_{\varepsilon, k}=\sqrt{1+4 \varepsilon \lambda_{k}}, \quad k=0,1, \ldots
$$

Lemma 5.2 Fix $\varepsilon \in(0,1 / 2]$. Then the $k$-th Fourier multiplier $b_{\varepsilon, k}$ of the operator $\mathcal{B}_{\varepsilon}$ is given by

$$
b_{\varepsilon, k}=\frac{3}{4} \frac{\left(X_{\varepsilon, k}+1\right)\left(X_{\varepsilon, k}^{2}+2 X_{\varepsilon, k}-1\right)}{X_{\varepsilon, k}+2} \sim 3 \varepsilon \lambda_{k} \quad(k \rightarrow+\infty) .
$$

Proof. Even if the proof can be obtained by arguing as in the proof of [4, Prop. 4.2], for the reader's convenience we provide the details.

The main step of the proof is the computation of the symbol of the operator $\varphi \mapsto u_{1}:=$ $\left(R\left(0, L_{\varepsilon}\right)\left[\varphi\left(\mathbf{V}-\mathbf{T}-\frac{4}{3} \mathbf{U}\right)\right]\right)_{1}(0, \cdot)$ for any $\varepsilon>0$. To lighten notation, throughout the proof we do not stress explicitly the dependence on $\varepsilon$ of the quantities we consider.

We claim that

$$
\widehat{u}_{1}(0, k)=-\frac{4}{9} \frac{4 X_{k}+7}{\left(X_{k}+1\right)^{2}\left(X_{k}+2\right)} \widehat{\varphi}(k), \quad k=0,1, \ldots
$$


Let us first assume that $\varphi$ is smooth enough. Since the function $\mathbf{V}-\mathbf{T}-\frac{4}{3} \mathbf{U}$ belongs to $(I-\mathcal{P})(X)$, from Theorem A.2 (iii) it follows that $\mathbf{u} \in D(L+\varepsilon A)$, so that $L \mathbf{u}+\varepsilon A \mathbf{u}=-\left(\mathbf{V}-\mathbf{T}-\frac{4}{3} \mathbf{U}\right) \varphi$. Moreover, the function $\widehat{\mathbf{u}}(\cdot, k)$ belongs to $(I-\mathcal{P})(D(L))$ and solves the equation $\left(\varepsilon \lambda_{k}-L\right) \widehat{\mathbf{u}}(\cdot, k)=$ $\left(\mathbf{V}-\mathbf{T}-\frac{4}{3} \mathbf{U}\right) \widehat{\varphi}(k)$ for any $k=0,1, \ldots$ Since $\lambda_{k}$ is in the resolvent set of the operator $L$ for any $k=0,1, \ldots$, by Theorem A.1 it follows that

$$
\widehat{\mathbf{u}}(\cdot, k)=R\left(\varepsilon \lambda_{k}, L\right)\left(\mathbf{V}-\mathbf{T}-\frac{4}{3} \mathbf{U}\right) \widehat{\varphi}(k), \quad k=0,1, \ldots
$$

Formula (5.4) can be extended to all functions $\varphi \in C_{\sharp}$ by a straightforward approximation argument.

From formula (A1) it is immediate to check that

$$
\begin{aligned}
\left(R\left(\varepsilon \lambda_{k}, L\right) \mathbf{f}\right)_{1}(0, \cdot)=\frac{1}{X_{k}}\left(\frac{4 \varepsilon \lambda_{k}}{\left(1+\left(2 \varepsilon \lambda_{k}-1\right) X_{k}\right)\left(X_{k}+1\right)}+1\right) \\
\times\left[\int_{-\infty}^{0} e^{-v_{1, k} t} f_{1}(t, \cdot) \mathrm{d} t+\int_{0}^{+\infty} e^{-\nu_{2, k} t} f_{2}(t, \cdot) \mathrm{d} t\right]
\end{aligned}
$$

for any $\mathbf{f}=\left(f_{1}, f_{2}\right) \in \mathcal{X}$, where

$$
v_{1, k}=\frac{1}{2}-\frac{1}{2} X_{k}, \quad v_{2, k}=\frac{1}{2}+\frac{1}{2} X_{k}, \quad k=0,1, \ldots
$$

Hence, from the definition of the functions $\mathbf{V}, \mathbf{T}$ and $\mathbf{U}$ (see 2.1) and 2.2), we get

$$
\begin{array}{r}
\int_{-\infty}^{0} e^{-v_{1, k} t}\left(V_{1}(t)-T_{1}(t)-\frac{4}{3} U_{1}(t)\right) \mathrm{d} t+\int_{0}^{+\infty} e^{-v_{2, k} t}\left(V_{2}(t)-T_{2}(t)-\frac{4}{3} U_{2}(t)\right) \mathrm{d} t \\
=-\frac{4}{9} \frac{\left(4 X_{k}+7\right)\left(X_{k}-1\right)}{\left(X_{k}+1\right)^{3}} .
\end{array}
$$

Since 0 is in the resolvent set of the restriction of $L$ to $(I-\mathcal{P})(\mathcal{X})$, we can extend the previous formula, by continuity, to $\lambda=0$. Thus,

$$
\begin{aligned}
\widehat{u}_{1}(0, k) & =-\frac{4}{9}\left(\frac{4 \varepsilon \lambda_{k}}{\left(1+\left(2 \varepsilon \lambda_{k}-1\right) X_{k}\right)\left(X_{k}+1\right)}+1\right) \frac{\left(4 X_{k}+7\right)\left(X_{k}-1\right)}{X_{k}\left(X_{k}+1\right)^{3}} \widehat{\varphi}(k) \\
& =-\frac{4}{9} \frac{4 X_{k}+7}{\left(X_{k}+2\right)\left(X_{k}+1\right)^{2}} \widehat{\varphi}(k)
\end{aligned}
$$

for any $k=0,1, \ldots$, and the assertion follows.

Now, using formula 5.3 it is immediate to complete the proof.

Proposition 5.3 For any $\varepsilon \in(0,1 / 2]$, the operator $\mathcal{B}_{\varepsilon}$ is invertible from $C_{\sharp}^{2+\theta}$ into $C_{\sharp}^{\theta}$ for any $\theta \in(0,1)$.

Proof. From Lemma 5.2, we know that $b_{\varepsilon, k} \neq 0$ for any $k=0,1, \ldots$ Hence, the operator $\mathcal{B}_{\varepsilon}$ admits a realization in $L^{2}$ which is invertible from $H^{2}$ into $L^{2}$. We still denote by $\mathcal{B}_{\varepsilon}$ such a realization. To prove that $\mathcal{B}_{\varepsilon}$ is invertible from $C_{\sharp}^{2}$ into $C_{\sharp}$, let us fix $f \in C_{\sharp}$ and let $u \in H^{2}$ be the unique solution to the equation $\mathcal{B}_{\varepsilon} u=f$. Taking [5.2] into account, it is immediate to check 
that we can split $\mathcal{B}_{\varepsilon}=-3 \varepsilon D_{\eta \eta}+\mathcal{B}_{\varepsilon}^{(1)}$, where $\mathcal{B}_{\varepsilon}^{(1)} \in L\left(H^{1}, L^{2}\right)$, since its symbol $\left(b_{\varepsilon, k}^{(1)}\right)$ satisfies

$$
b_{\varepsilon, k}^{(1)} \sim \frac{3}{2} \sqrt{\varepsilon \lambda_{k}} \quad(k \rightarrow+\infty) .
$$

It follows that the function $\mathcal{B}_{\varepsilon}^{(1)}(u)$ is in $C_{\sharp}^{1 / 2}$. Consequently, $u_{\eta \eta}$ is in $C_{\sharp}$.

Let us now suppose that $f \in C_{\sharp}^{\theta}$ with $\theta \leqslant 1 / 2$ and let $u \in H^{2}$ satisfy $\mathcal{B}_{\varepsilon}(u)=f$. Since $\mathcal{B}_{\varepsilon}^{(1)}(u) \subset C_{\sharp}^{1 / 2}, u_{\eta \eta}$ belongs to $C_{\sharp}^{\theta}$ and hence $u \in C_{\sharp}^{2+\theta}$. Let now $\theta \in(1 / 2,1)$. The above result shows that $u \in C_{\sharp}^{5 / 2}$. Since $C_{\sharp}^{5 / 2} \subset H^{5 / 2}, \mathcal{B}_{\varepsilon}^{(1)} u \in H^{3 / 2} \subset C_{\sharp}^{\theta}$. Hence, also in this case $u_{\eta \eta} \in C_{\sharp}^{\theta}$ and we are done.

In view of Proposition 5.3 , we can invert the operator $\mathcal{B}_{\varepsilon}$ from $C_{\sharp}^{2+\theta}$ into $C_{\sharp}^{\theta}$ for any $\theta \in(0,1)$, getting the following equation for $\rho$ :

$$
\rho_{\tau}(\tau, \cdot)=\mathcal{R}_{\varepsilon}(\rho(\tau, \cdot))+\mathcal{K}_{\varepsilon}\left(\tau, \rho_{\eta}(\tau, \cdot)\right), \quad \tau \in[0, T],
$$

where

$$
\begin{aligned}
\mathcal{R}_{\varepsilon}(\rho)= & \mathcal{B}_{\varepsilon}^{-1}\left(\mathcal{S}_{\varepsilon}(\rho)\right) \\
\mathcal{K}_{\varepsilon}(\tau, \rho)= & \mathcal{B}_{\varepsilon}^{-1}\left(\mathcal{G}_{\varepsilon}\left(\left(\Phi_{\eta}(\tau, \cdot)\right)^{2}\right)\right)-\mathcal{B}_{\varepsilon}^{-1}\left(\Phi_{\eta}(\tau, \cdot) \rho\right)+2 \varepsilon \mathcal{B}_{\varepsilon}^{-1}\left(\mathcal{G}_{\varepsilon}\left(\Phi_{\eta}(\tau, \cdot) \rho\right)\right) \\
& -\frac{\varepsilon}{2} \mathcal{B}_{\varepsilon}^{-1}\left(\rho^{2}\right)+\varepsilon^{2} \mathcal{B}_{\varepsilon}^{-1}\left(\mathcal{G}_{\varepsilon}\left(\rho^{2}\right)\right)+3 \mathcal{B}_{\varepsilon}^{-1}\left(\Phi_{\eta \eta \eta \eta}\right) \\
& +3 \mathcal{B}_{\varepsilon}^{-1}\left(\left(D_{\eta \eta} R\left(0, L_{\varepsilon}\right)\left[\Phi_{\eta \eta}\left(\mathbf{T}^{\prime}-\mathbf{U}\right)\right]\right)_{1}(\cdot, 0, \cdot)\right)+\frac{13}{3} \mathcal{B}_{\varepsilon}^{-1}\left(\Phi_{\tau \eta \eta}\right) \\
& -3 \varepsilon \mathcal{B}_{\varepsilon}^{-1}\left(\left(D_{\eta \eta} R\left(0, L_{\varepsilon}\right)\left[\Phi_{\tau \eta \eta}\left(\mathbf{V}-\mathbf{T}-\frac{4}{3} \mathbf{U}\right)\right]\right)_{1}(\cdot, 0, \cdot)\right) .
\end{aligned}
$$

\subsection{Solving equation 5.5 ,}

Here, we prove an existence and uniqueness result for equation 5.5 with initial condition $\rho(0, \cdot)=0$. For this purpose, we need to thoroughly study the operators $\mathcal{R}_{\varepsilon}$ and $\mathcal{K}_{\varepsilon}$. To lighten notation, we do not stress explicitly the dependence on $\varepsilon$ of the symbols of the operators we are going to consider. In particular, we simply write $X_{k}$ for $X_{\varepsilon, k}$ (see (5.1)).

We begin by considering the operator $\mathcal{R}_{\varepsilon}$. Taking Proposition 5.3 and Theorem A.2 (iii) into account, it is immediate to check that $\mathcal{R}_{\varepsilon}$ is well-defined in $C_{\sharp}^{4}$. Actually, we show that it can be extended to $C_{\sharp}^{1} \cap C^{2}$ as a bounded operator which is sectorial.

PROPOSITION 5.4 For any $\varepsilon \in(0,1 / 2]$, the operator $\mathcal{R}_{\varepsilon}$ can be extended to a sectorial operator $R_{\varepsilon}$ with domain $C_{\sharp}^{1} \cap C^{2}$. Moreover, $D_{R_{\varepsilon}}(\theta, \infty)=C_{\sharp}^{2 \theta}$ for any $\theta \in(0,1) \backslash\{1 / 2\}$, with equivalence of the corresponding norms.

Proof. To begin, we compute the symbol of the operator $\mathcal{S}_{\varepsilon}$ (see (4.17b)). For this purpose we set $v_{1}:=\left(R\left(0, L_{\varepsilon}\right)\left[\varphi\left(\mathbf{T}^{\prime}-\mathbf{U}\right)\right]\right)_{1}(0, \cdot)$ and observe that, arguing as in the proof of $[5.3$, we can easily show that

$$
\widehat{v}_{1}(0, k)=\frac{2}{3} \frac{1}{\left(X_{k}+1\right)\left(X_{k}+2\right)} \widehat{\varphi}(k), \quad k=0,1, \ldots
$$


Hence, we have

$$
s_{k}=\frac{3\left(X_{k}-1\right)\left(X_{k}+1\right)^{2}\left\{(\varepsilon-1) X_{k}^{2}+(\varepsilon-1) X_{k}+2\right\}}{16 \varepsilon^{2}\left(X_{k}+2\right)} \sim 48(\varepsilon-1) \varepsilon^{2} \lambda_{k}^{2}
$$

as $k \rightarrow+\infty$. From (5.2) and 5.8 it follows that the $k$-th symbol of the operator $\mathcal{R}_{\varepsilon}$ is

$$
r_{k}=\frac{\left(X_{k}^{2}-1\right)\left\{(\varepsilon-1) X_{k}^{2}+(\varepsilon-1) X_{k}+2\right\}}{4 \varepsilon^{2}\left(X_{k}^{2}+2 X_{k}-1\right)}, \quad k=0,1, \ldots
$$

For any fixed $\varepsilon \in(0,1 / 2], r_{k} \sim\left(1-\varepsilon^{-1}\right) \lambda_{k}$ as $k \rightarrow+\infty$. Hence, we can split

$$
\mathcal{R}_{\varepsilon} \varphi=\frac{1-\varepsilon}{\varepsilon} \varphi_{y y}+\mathcal{R}_{\varepsilon}^{(1)} \varphi
$$

where the symbol of $\mathcal{R}_{\varepsilon}^{(1)}$ is

$$
r_{k}^{(1)}=\frac{\left(X_{k}^{2}-1\right)\left\{(1-\varepsilon) X_{k}+\varepsilon+1\right\}}{4 \varepsilon^{2}\left(X_{k}^{2}+2 X_{k}-1\right)} \sim \frac{(1-\varepsilon) \sqrt{\varepsilon}}{2 \varepsilon^{2}} \sqrt{\lambda_{k}} \quad(k \rightarrow+\infty) .
$$

We claim that the operator $\mathcal{R}_{\varepsilon}^{(1)}$ admits a realization in $C^{0}$ which is in $L\left(C_{\sharp}^{1+\alpha}, C_{\sharp}\right)$ for any $\alpha \in$ $(1 / 2,1)$. As a first step, we observe that, due to the characterization of the spaces $H^{s}$ given in 2.3, the operator $\mathcal{R}_{\varepsilon}^{(1)}$ admits a realization $R_{\varepsilon}^{(1)} \in L\left(H^{s}, H^{s-1}\right)$ for any $s \geqslant 1$. It is well-known that $C_{\sharp}^{m} \subset H^{m} \subset C_{\sharp}^{m-1 / 2}$ with continuous embeddings for any $m>1 / 2$ such that $m-1 / 2 \notin \mathbb{N}$. As a consequence, $R_{\varepsilon}^{(1)} \in L\left(C_{\sharp}^{s}, C_{\sharp}^{s-3 / 2}\right)$ for any $s>3 / 2$ such that $s-3 / 2 \notin \mathbb{N}$. Therefore, $\mathcal{R}_{\varepsilon}$ can be extended with a bounded operator $R_{\varepsilon}$ from $D\left(R_{\varepsilon}\right)=C_{\sharp}^{1} \cap C^{2}$ into $C^{0}$.

Let us now prove that $R_{\varepsilon}$ is sectorial. For this purpose, we note that $C_{\sharp}^{\theta}$ belongs to the class $J_{\theta / 2}$ between $C^{0}$ and $C_{\sharp}^{1} \cap C^{2}$, for any $\theta \in(0,2)$, i.e., there exists a positive constant $K$ such that

$$
\|f\|_{C^{\theta}} \leqslant K\|f\|_{C^{0}}^{(2-\theta) / 2}\|f\|_{C^{2}}^{\theta / 2}
$$

for any $f \in C_{\sharp}^{1} \cap C^{2}$, and the realization of the second-order derivative in $C\left(\left[-\ell_{0} / 2, \ell_{0} / 2\right]\right)$ with domain $C_{\sharp}^{1} \cap C^{2}$ is sectorial. Hence, we can apply [11, Prop. 2.4.1(i)] to conclude that the operator $R_{\varepsilon}$ is sectorial in $C^{0}$. It is now clear that the graph norm of $R_{\varepsilon}$ is equivalent to the Euclidean norm of $C_{\sharp}^{1} \cap C^{2}$. Hence, [11, Prop. 2.2.2] implies that $D_{R_{\varepsilon}}(\theta, \infty)=C_{\sharp}^{2 \theta}$ for any $\theta \in(0,1) \backslash\{1 / 2\}$.

We now consider the operator $\mathcal{K}_{\varepsilon}$ of (5.6). From Proposition 5.3 and Theorems 4.4 and A.2 (iii), we know that $\mathcal{K}_{\varepsilon}$ is continuous from $C_{\sharp}^{2+\alpha}$ into $[0,+\infty) \times C_{\sharp}$ for any $\alpha>0$. Let us show that it can be extended to a larger domain.

Proposition 5.5 Let the assumptions of Theorem 4.4 be satisfied. Then, for any $\varepsilon \in(0,1 / 2]$, the operator $\mathcal{K}_{\varepsilon}$ can be extended to a continuous operator mapping $C_{\sharp}^{S}$ into $[0,+\infty) \times C_{\sharp}^{S}$ for any $s \geqslant 0$. Moreover, for any $r, T>0$, there exists a positive constant $K=K(T, r)$ such that

$$
\left\|\mathcal{K}_{\varepsilon}\left(\tau_{2}, \psi\right)-\mathcal{K}_{\varepsilon}\left(\tau_{1}, \psi\right)\right\|_{\infty}+\left\|\mathcal{K}_{\varepsilon}(\tau, \psi)-\mathcal{K}_{\varepsilon}(\tau, \xi)\right\|_{\infty} \leqslant K\left(\left|\tau_{2}-\tau_{1}\right|+\|\psi-\xi\|_{\infty}\right)
$$

for any $\tau, \tau_{1}, \tau_{2} \in[0, T]$ and any $\psi, \xi \in B(0, r) \subset C_{\sharp}$. 
Proof. As a first step, we observe that, using formulae (5.3) and (5.7), one can easily show that the $k$-th symbol $g_{k}$ of the operator $\mathcal{G}_{\varepsilon}$ is

$$
g_{k}=-\lambda_{k} \frac{3 X_{k}^{2}+15 X_{k}+4}{2\left(X_{k}+1\right)\left(X_{k}+2\right)} .
$$

From 5.2 and 5.10 , it follows that the symbol of the operator $\mathcal{Z}_{\varepsilon}:=\mathcal{B}_{\varepsilon}^{-1} \mathcal{G}_{\varepsilon}$ is

$$
z_{k}=-\frac{2}{3} \lambda_{k} \frac{3 X_{k}^{2}+15 X_{k}+4}{\left(X_{k}+1\right)^{2}\left(X_{k}^{2}+2 X_{k}-1\right)}=-\frac{1}{2 \varepsilon}+z_{k}^{(1)},
$$

where

$$
z_{k}^{(1)} \sim-\frac{1}{4 \sqrt{\varepsilon^{3} \lambda_{k}}}, \quad k \rightarrow+\infty
$$

We can thus split

$$
z_{\varepsilon}=-\frac{1}{2 \varepsilon} \mathrm{Id}+Z_{\varepsilon}^{(1)}
$$

where $z_{\varepsilon}^{(1)}$ is the operator whose symbol is $\left(z_{k}^{(1)}\right)$. Formula 5.11) shows that $z_{\varepsilon}^{(1)} \in L\left(H^{s}, H^{s+1}\right)$ for any $s \geqslant 0$. Hence, $Z_{\varepsilon}^{(1)} \in L\left(C_{\sharp}^{s}, C_{\sharp}^{s+\theta}\right)$ for any $s \in \mathbb{N} \cup\{0\}$ and any $\theta \in(0,1 / 2)$. As a byproduct, $Z_{\varepsilon} \in L\left(C_{\sharp}^{S}\right)$ for any $s \geqslant 0$. The assertion now follows from (5.6) on taking Proposition 5.3 and Theorem 4.4 into account.

From all the previous results, we get the following:

TheOREM 5.6 For any $\varepsilon \in(0,1 / 2]$, equation 5.5 admits a unique solution $\rho$, defined in a maximal time domain $\left[0, T_{\varepsilon}\right)$, which vanishes at $\tau=0$, belongs to $C^{1,2}\left(\left[0, T_{\varepsilon}\right) \times\left[-\ell_{0} / 2, \ell_{0} / 2\right]\right)$ and satisfies $D_{\eta}^{(j)} \rho\left(\cdot,-\ell_{0} / 2\right) \equiv D_{\eta}^{(j)} \rho\left(\cdot, \ell_{0} / 2\right)$ for $j=0,1$.

Proof. Combining [11, Thms. 7.1.2 \& 4.3.8], we can easily show that equation (5.5) admits a unique solution $\rho$, defined in a maximal time domain $\left[0, T_{\varepsilon}\right)$, which belongs to $C^{1, \beta}\left(\left[0, T_{\varepsilon}\right) \times\right.$ $\left.\left[-\ell_{0} / 2, \ell_{0} / 2\right]\right)$ for any $\beta<2$, vanishes at $\tau=0$, and satisfies $D_{\eta}^{(j)} \rho\left(\cdot,-\ell_{0} / 2\right) \equiv D_{\eta}^{(j)} \rho\left(\cdot, \ell_{0} / 2\right)$ for $j=0,1$. Moreover, $R_{\varepsilon}(\rho)$ is continuous in $\left[0, T_{\varepsilon}\right) \times\left[-\ell_{0} / 2, \ell_{0} / 2\right]$. Since $R_{\varepsilon}(\rho)$ and $\frac{1-\varepsilon}{\varepsilon} D_{\eta \eta}$ differ by the the lower order operator $R_{\varepsilon}^{(1)}$ (see the proof of Proposition 5.4, $\rho_{\eta \eta} \in C\left(\left[0, T_{\varepsilon}\right) \times\right.$ $\left.\left[-\ell_{0} / 2, \ell_{0} / 2\right]\right)$ as well, and this completes the proof.

\subsection{Proof of Theorem 5.1}

This subsection is the final step of the proof of Theorem 5.1. Using some bootstrap arguments, we show that the solution $\rho$ to equation 5.5 given by Theorem 5.6 is actually a solution to equation 4.20]. Of course, we just need to show that both the functions $\rho_{\eta \eta}$ and $\rho_{\tau}$ belong to $C^{0,2}\left(\left[0, T_{\varepsilon}\right) \times\right.$ $\left.\left[-\ell_{0} / 2, \ell_{0} / 2\right]\right)$. Throughout the proof, we assume that $T^{\prime}$ is any fixed number in the interval $\left[0, T_{\varepsilon}\right)$.

To begin, we observe that Propositions 5.4 and 5.5 show that $\mathcal{K}_{\varepsilon}(\cdot, \rho) \in C\left(\left[0, T^{\prime}\right] ; D_{R_{\varepsilon}}(\theta, \infty)\right)$ for any $\theta \in(0,1)$. Therefore, we can apply [11, Thm. 4.3.8] and conclude that $\rho_{\tau}, R_{\varepsilon}(\rho)$ are bounded in $\left[0, T^{\prime}\right]$ with values in $C_{\sharp}^{2 \theta}$ for any $\theta \in(0,1)$. Since $R_{\varepsilon}(\rho)$ and $\frac{1-\varepsilon}{\varepsilon} D_{\eta \eta}$ differ by the lower order operator $R_{\varepsilon}^{(1)}, \rho_{\eta \eta} \in C^{0,2 \theta}\left(\left[0, T^{\prime}\right] \times\left[-\ell_{0} / 2, \ell_{0} / 2\right]\right)$ for any $\theta$ as above. In particular, $\rho_{\tau}$ and $\rho_{\eta \eta}$ belong to $C^{0,1}\left(\left[0, T^{\prime}\right] \times\left[-\ell_{0} / 2, \ell_{0} / 2\right]\right)$. 
Let us now set $\zeta:=\rho_{\eta}$. Clearly, $\zeta \in C\left(\left[0, T_{\varepsilon}\right) ; C_{\sharp}^{2}\right) \cap C^{1}\left(\left[0, T_{\varepsilon}\right) ; C_{\sharp}\right)$ and $\zeta_{\tau}=\rho_{\eta \tau}$. Moreover,

$$
\zeta_{\tau}=\left(1-\varepsilon^{-1}\right) \zeta_{\eta \eta}+D_{\eta} R_{\varepsilon}^{(1)} \rho+D_{\eta} \mathcal{K}_{\varepsilon}\left(\cdot, \rho_{\eta}\right)
$$

in $\left[0, T_{\varepsilon}\right) \times\left[-\ell_{0} / 2, \ell_{0} / 2\right]$, and $\zeta(0, \cdot) \equiv 0$. Since $R_{\varepsilon}^{(1)} \in L\left(C_{\sharp}^{3+\theta}, C_{\sharp}^{3 / 2+\theta}\right)$ for any $\theta \in(0,1) \backslash\{1 / 2\}$, the function $D_{\eta} R_{\varepsilon}^{(1)}(\rho)$ is bounded in $\left[0, T^{\prime}\right]$ with values in $C_{\sharp}^{\alpha}$ for any $\alpha<3 / 2$. Using Proposition 5.5 we now deduce that $D_{\eta} R_{\varepsilon}^{(1)}(\rho)+D_{\eta} \mathcal{K}_{\varepsilon}\left(\cdot, \rho_{\eta}(\tau, \cdot)\right)$ is bounded in $\left[0, T^{\prime}\right]$ with values in $C_{\sharp}^{\alpha}$ for any $\alpha$ as above. Hence, Theorem 4.3.9(iii) of [11] implies that the functions $\zeta_{\tau}$ and $\zeta_{\eta \eta}$ are bounded (in fact, continuous) in $\left[0, T^{\prime}\right]$ with values in $C_{\sharp}^{\alpha}$. This completes the proof.

\section{Uniform existence of $\rho$ and proof of the main result}

So far we have only proved a local existence-uniqueness result for equation (4.20) on an $\varepsilon$-dependent time interval. In this section, we want to prove that, for any fixed $T>0$, the local solution $\rho$ exists in the whole of $[0, T]$, at least for sufficiently small values of $\varepsilon$. The main tool in this direction is represented by the a priori estimates in the next subsection.

\subsection{A priori estimates}

The main result of this subsection is contained in the following theorem.

Theorem 6.1 For any $T>0$, there exist $\varepsilon_{0}=\varepsilon_{0}(T) \in(0,1 / 2)$ and $K=K(T)>0$ such that, for any $T^{\prime}<T$ and any $\rho \in y_{T^{\prime}}$ (see Definition 4.1) which solves equation 4.20), we have

$$
\begin{aligned}
& \sup _{\substack{\tau \in\left(0, T^{\prime}\right] \\
\eta \in\left[-\ell_{0} / 2, \ell_{0} / 2\right]}}\left|\rho_{\eta}(\tau, \eta)\right|+\sup _{\tau \in\left(0, T^{\prime}\right]} \int_{-\ell_{0} / 2}^{\ell_{0} / 2}\left(\rho_{\eta \eta}(\tau, \eta)\right)^{2} \mathrm{~d} \eta \\
& \\
& +\int_{0}^{T^{\prime}} \int_{-\ell_{0} / 2}^{\ell_{0} / 2}\left(\rho_{\tau}(\tau, \eta)\right)^{2} \mathrm{~d} \eta \mathrm{d} \tau+\int_{0}^{T^{\prime}} \int_{-\ell_{0} / 2}^{\ell_{0} / 2}\left(\rho_{\tau \eta}(\tau, \eta)\right)^{2} \mathrm{~d} \eta \mathrm{d} \tau \leqslant K
\end{aligned}
$$

for all $\tau \in\left(0, T^{\prime}\right]$, whenever $\varepsilon \leqslant \varepsilon_{0}$.

The proof of Theorem 6.1 employs an energy method. Let $\rho \in y_{T^{\prime}}$ solve 4.20), i.e.,

$$
\frac{\partial}{\partial \tau} \mathcal{B}_{\varepsilon}(\rho)=\mathcal{S}_{\varepsilon}(\rho)-\Phi_{\eta} \rho_{\eta}-\frac{\varepsilon}{2}\left(\rho_{\eta}\right)^{2}+\mathcal{G}_{\varepsilon}\left(\left(\Phi_{\eta}+\varepsilon \rho_{\eta}\right)^{2}\right)+\mathcal{H}_{\varepsilon}(\Phi) .
$$

Multiplying both sides of (6.1) by $\rho_{\tau}$ and integrating over $\left[-\ell_{0} / 2, \ell_{0} / 2\right]$, we get

$$
\begin{aligned}
\int_{-\ell_{0} / 2}^{\ell_{0} / 2} \mathcal{B}_{\varepsilon}\left(\rho_{\tau}\right) \rho_{\tau} \mathrm{d} \eta= & \int_{-\ell_{0} / 2}^{\ell_{0} / 2} \mathcal{S}_{\varepsilon}(\rho) \rho_{\tau} \mathrm{d} \eta-\int_{-\ell_{0} / 2}^{\ell_{0} / 2} \Phi_{\eta} \rho_{\eta} \rho_{\tau} \mathrm{d} \eta-\frac{\varepsilon}{2} \int_{-\ell_{0} / 2}^{\ell_{0} / 2}\left(\rho_{\eta}\right)^{2} \rho_{\tau} \mathrm{d} \eta \\
& +\int_{-\ell_{0} / 2}^{\ell_{0} / 2} \mathcal{G}_{\varepsilon}\left(\left(\Phi_{\eta}+\varepsilon \rho_{\eta}\right)^{2}\right) \rho_{\tau} \mathrm{d} \eta+\int_{-\ell_{0} / 2}^{\ell_{0} / 2} \mathcal{H}_{\varepsilon}(\Phi) \rho_{\tau} \mathrm{d} \eta .
\end{aligned}
$$


Using the definition of the operator $\mathcal{S}_{\varepsilon}$ (see 4.17b) and then integrating by parts yields

$$
\begin{aligned}
\int_{-\ell_{0} / 2}^{\ell_{0} / 2} \mathcal{S}_{\varepsilon}(\rho) \rho_{\tau} \mathrm{d} \eta \\
=\int_{-\ell_{0} / 2}^{\ell_{0} / 2}\left\{-3(1-\varepsilon) \rho_{\eta \eta \eta \eta}-\rho_{\eta \eta}+3 \varepsilon\left(D_{\eta \eta} R\left(0, L_{\varepsilon}\right)\left[\rho_{\eta \eta}\left(\mathbf{T}^{\prime}-\mathbf{U}\right)\right]\right)_{1}(\cdot, 0, \cdot)\right\} \rho_{\tau} \mathrm{d} \eta \\
=-\frac{3}{2}(1-\varepsilon) \frac{\mathrm{d}}{\mathrm{d} t} \int_{-\ell_{0} / 2}^{\ell_{0} / 2}\left(\rho_{\eta \eta}\right)^{2} \mathrm{~d} \eta-\int_{-\ell_{0} / 2}^{\ell_{0} / 2} \rho_{\eta \eta} \rho_{\tau} \mathrm{d} \eta \\
\quad+3 \varepsilon \int_{-\ell_{0} / 2}^{\ell_{0} / 2}\left(D_{\eta \eta} R\left(0, L_{\varepsilon}\right)\left[\rho_{\eta \eta}\left(\mathbf{T}^{\prime}-\mathbf{U}\right)\right]\right)_{1}(\cdot, 0, \cdot) \rho_{\tau} \mathrm{d} \eta .
\end{aligned}
$$

Therefore, we can write equation 6.2 in the following equivalent form:

$$
\begin{aligned}
\frac{3}{2}(1-\varepsilon) & \frac{\mathrm{d}}{\mathrm{d} \tau} \int_{-\ell_{0} / 2}^{\ell_{0} / 2}\left(\rho_{\eta \eta}\right)^{2} \mathrm{~d} \eta+\int_{-\ell_{0} / 2}^{\ell_{0} / 2} \mathcal{B}_{\varepsilon}\left(\rho_{\tau}\right) \rho_{\tau} \mathrm{d} \eta \\
= & -\frac{\varepsilon}{2} \int_{-\ell_{0} / 2}^{\ell_{0} / 2}\left(\rho_{\eta}\right)^{2} \rho_{\tau} \mathrm{d} \eta-\int_{-\ell_{0} / 2}^{\ell_{0} / 2} \rho_{\eta \eta} \rho_{\tau} \mathrm{d} \eta \\
& +3 \varepsilon \int_{-\ell_{0} / 2}^{\ell_{0} / 2}\left(D_{\eta \eta} R\left(0, L_{\varepsilon}\right)\left[\rho_{\eta \eta}\left(\mathbf{T}^{\prime}-\mathbf{U}\right)\right]\right)_{1}(\cdot, 0, \cdot) \rho_{\tau} \mathrm{d} \eta \\
& +\int_{-\ell_{0} / 2}^{\ell_{0} / 2} \mathcal{G}_{\varepsilon}\left(\left(\Phi_{\eta}+\varepsilon \rho_{\eta}\right)^{2}\right) \rho_{\tau} \mathrm{d} \eta-\int_{-\ell_{0} / 2}^{\ell_{0} / 2} \Phi_{\eta} \rho_{\eta} \rho_{\tau} \mathrm{d} \eta+\int_{-\ell_{0} / 2}^{\ell_{0} / 2} \mathcal{H}_{\varepsilon}(\Phi) \rho_{\tau} \mathrm{d} \eta .
\end{aligned}
$$

In the following lemmata we estimate the terms

$$
\begin{aligned}
\mathcal{J}_{1} & :=\int_{-\ell_{0} / 2}^{\ell_{0} / 2} \mathcal{B}_{\varepsilon}\left(\rho_{\tau}\right) \rho_{\tau} \mathrm{d} \eta \\
\mathcal{J}_{2} & :=\int_{-\ell_{0} / 2}^{\ell_{0} / 2}\left(D_{\eta \eta} R\left(0, L_{\varepsilon}\right)\left[\rho_{\eta \eta}\left(\mathbf{T}^{\prime}-\mathbf{U}\right)\right]\right)_{1}(\cdot, 0, \cdot) \rho_{\tau} \mathrm{d} \eta \\
\mathcal{J}_{3} & :=\int_{-\ell_{0} / 2}^{\ell_{0} / 2} \mathcal{G}_{\varepsilon}\left(\left(\Phi_{\eta}+\varepsilon \rho_{\eta}\right)^{2}\right) \rho_{\tau} \mathrm{d} \eta \\
\mathcal{J}_{4} & :=\int_{-\ell_{0} / 2}^{\ell_{0} / 2} \mathcal{H}_{\varepsilon}(\Phi) \rho_{\tau} \mathrm{d} \eta .
\end{aligned}
$$

The main issue is to control $\mathcal{J}_{1}$ :

LEMMA 6.2 We have

$$
\mathcal{J}_{1}(\tau) \geqslant \int_{-\ell_{0} / 2}^{\ell_{0} / 2}\left(\rho_{\tau}(\tau, \cdot)\right)^{2} \mathrm{~d} \eta+3 \varepsilon \int_{-\ell_{0} / 2}^{\ell_{0} / 2}\left(\rho_{\tau \eta}(\tau, \cdot)\right)^{2} \mathrm{~d} \eta
$$

for any $\tau \in\left[0, T^{\prime}\right]$ and any $\varepsilon \in(0,1 / 2]$. 
Proof. Of course, we can limit ourselves to proving the estimate with $\rho_{\tau}(\tau, \cdot)$ replaced by $\varphi \in H^{2}$. It is immediate to check that

$$
\int_{-\ell_{0} / 2}^{\ell_{0} / 2} \mathcal{B}_{\varepsilon}(\varphi) \varphi \mathrm{d} \eta=\sum_{k=0}^{+\infty} b_{\varepsilon, k}|\widehat{\varphi}(k)|^{2}
$$

where the symbol $\left(b_{\varepsilon, k}\right)$ of the operator $\mathcal{B}_{\varepsilon}$ is defined by $(5.2)$. Note that $b_{\varepsilon, k}=h\left(X_{\varepsilon, k}\right)$ for any $k=0,1, \ldots$, where the function $h:[1,+\infty) \rightarrow \mathbb{R}$ is defined by

$$
h(s)=\frac{3}{4} \frac{(s+1)\left(s^{2}+2 s-1\right)}{s+2}, \quad s \geqslant 1 .
$$

Since $h(s) \geqslant\left(3 s^{2}+1\right) / 4$ for any $s \geqslant 1$, we can estimate

$$
\int_{-\ell_{0} / 2}^{\ell_{0} / 2} \mathcal{B}_{\varepsilon}(\varphi) \varphi \mathrm{d} \eta \geqslant \sum_{k=0}^{+\infty}\left(1+3 \varepsilon \lambda_{k}\right)|\widehat{\varphi}(k)|^{2}=\int_{-\ell_{0} / 2}^{\ell_{0} / 2}|\varphi|^{2} \mathrm{~d} \eta+3 \varepsilon \int_{-\ell_{0} / 2}^{\ell_{0} / 2}\left|\varphi_{\eta}\right|^{2} \mathrm{~d} \eta,
$$

and we are done.

We now consider the terms $\mathcal{J}_{2}, \mathcal{J}_{3}$ and $\mathcal{J}_{4}$.

LEMMA 6.3 For any $\tau \in\left[0, T^{\prime}\right]$ and any $\varepsilon \in(0,1 / 2]$,

$$
\left|\mathcal{J}_{2}(\tau)\right| \leqslant \frac{1}{12 \varepsilon}\left\|\rho_{\tau}(\tau, \cdot)\right\|_{2}\left\|\rho_{\eta \eta}(\tau, \cdot)\right\|_{2} .
$$

Proof. It is immediately seen that for any $\tau \in[0, T]$ we can estimate

$$
\begin{aligned}
& \left|\int_{-\ell_{0} / 2}^{\ell_{0} / 2}\left(D_{\eta \eta} R\left(0, L_{\varepsilon}\right)\left[\rho_{\eta \eta}\left(\mathbf{T}^{\prime}-\mathbf{U}\right)\right]\right)_{1}(\tau, 0, \cdot) \rho_{\tau}(\tau, \cdot) \mathrm{d} \eta\right| \\
& \leqslant \frac{1}{3 \varepsilon}\left\|\varrho_{\tau}(\tau, \cdot)\right\|_{2}\left\|\left(D_{\eta \eta} R\left(0, L_{\varepsilon}\right)\left[\rho_{\eta \eta}\left(\mathbf{T}^{\prime}-\mathbf{U}\right)\right]\right)_{1}(\tau, 0, \cdot)\right\|_{2} .
\end{aligned}
$$

To compute the $L^{2}$-norm of the function $\left(D_{\eta \eta} R\left(0, L_{\varepsilon}\right)\left[\rho_{\eta \eta}\left(\mathbf{T}^{\prime}-\mathbf{U}\right)\right]\right)_{1}(\tau, 0, \cdot)$, we take advantage of formula (5.7), which allows us to estimate

$$
\left\|\left(D_{\eta \eta} R\left(0, L_{\varepsilon}\right)\left[\rho_{\eta \eta}\left(\mathbf{T}^{\prime}-\mathbf{U}\right)\right]\right)_{1}(\tau, 0, \cdot)\right\|_{2}^{2}=\frac{1}{4} \sum_{k=0}^{+\infty}\left|\frac{X_{k}+1}{X_{k}+2} \lambda_{k}^{2} \widehat{\rho}(\tau, k)\right|^{2} \leqslant \frac{1}{4}\left\|\rho_{\eta \eta}(\tau, \cdot)\right\|_{2}^{2},
$$

where, as usual, $X_{k}=\sqrt{1+4 \varepsilon \lambda_{k}}$ and $\widehat{\rho}(\tau, k)$ is the $k$-th Fourier coefficient of the function $\rho(\tau, \cdot)$. This accomplishes the proof.

LEMmA 6.4 There exists a positive constant $C$, independent of $\varepsilon \in(0,1 / 2], \tau$ and $T^{\prime}$, such that

$$
\begin{aligned}
\left|\mathcal{J}_{3}(\tau)\right| \leqslant & C\left(\left\|\rho_{\tau}(\tau, \cdot)\right\|_{2}+\left\|\rho_{\tau}(\tau, \cdot)\right\|_{2}\left\|\rho_{\eta \eta}(\tau, \cdot)\right\|_{2}+\varepsilon\left\|\rho_{\tau}(\tau, \cdot)\right\|_{2}\left\|\rho_{\eta \eta}(\tau, \cdot)\right\|_{2}^{2}\right. \\
& \left.+\varepsilon\left\|\rho_{\tau \eta}(\tau, \cdot)\right\|_{2}\left\|\rho_{\eta \eta}(\tau, \cdot)\right\|_{2}+\varepsilon^{2}\left\|\rho_{\tau \eta}(\tau, \cdot)\right\|_{2}\left\|\rho_{\eta \eta}(\tau, \cdot)\right\|_{2}^{2}\right)
\end{aligned}
$$

for any $\tau \in\left[0, T^{\prime}\right]$ 
Proof. As in the proof of the previous lemma, it is enough to estimate the $L^{2}$-norm of the function $\mathcal{G}_{\varepsilon}\left(\left(\Phi_{\eta}(\tau, \cdot)+\varepsilon \rho_{\eta}(\tau, \cdot)\right)^{2}\right)$. For this purpose, we observe that (see $[5.10)$

$$
\left\|\mathcal{G}_{\varepsilon}(\psi)\right\|_{2}^{2}=\sum_{k=0}^{+\infty} \lambda_{k}^{2}\left(\frac{3 X_{k}^{2}+15 X_{k}+4}{2\left(X_{k}+1\right)\left(X_{k}+2\right)}\right)^{2}|\widehat{\psi}(k)|^{2} \leqslant \frac{121}{36} \sum_{k=0}^{+\infty} \lambda_{k}^{2}|\widehat{\psi}(k)|^{2}
$$

for any $\psi \in H^{2}$, where $X_{k}=\sqrt{1+4 \varepsilon \lambda_{k}}$ for any $k=0,1, \ldots$ It follows that

$$
\left\|\mathcal{G}_{\varepsilon}(\psi)\right\|_{2} \leqslant 2\left\|\psi_{\eta \eta}\right\|_{2}
$$

Moreover, the symbol $g_{k}$ can be split as follows:

$$
g_{k}=-\frac{3}{2} \lambda_{k}+\frac{1}{4 \varepsilon} h\left(X_{k}\right), \quad k=0,1, \ldots,
$$

where the function $h:[1,+\infty) \rightarrow \mathbb{R}$ is defined by

$$
h(s)=\frac{(1-3 s)(s-1)}{s+2}, \quad s \geqslant 1 .
$$

Clearly, $-3 s \leqslant h(s) \leqslant 0$ for any $s \geqslant 1$. Hence, we can split

$$
\mathcal{G}_{\varepsilon}(\psi)=\frac{3}{2} \psi_{\eta \eta}+\frac{1}{4 \varepsilon} \mathcal{G}_{\varepsilon}^{(1)}(\psi)
$$

where $\mathcal{G}_{\varepsilon}^{(1)} \in L\left(H^{1}, L^{2}\right)$ and

$$
\left\|\mathcal{G}_{\varepsilon}^{(1)}(\psi)\right\|_{2} \leqslant 3\left(\sum_{k=0}^{+\infty}\left(1+4 \varepsilon \lambda_{k}\right)|\widehat{\psi}(k)|^{2}\right)^{1 / 2}=3\left(\|\psi\|_{2}^{2}+4 \varepsilon\left\|\psi_{\eta}\right\|_{2}^{2}\right)^{1 / 2} \leqslant 6\|\psi\|_{H^{1}}
$$

We now split (for any arbitrarily fixed $\tau \in\left[0, T^{\prime}\right]$ )

$$
\begin{aligned}
& \int_{-\ell_{0} / 2}^{\ell_{0} / 2} \mathcal{G}_{\varepsilon}\left(\left(\Phi_{\eta}(\tau, \cdot)+\varepsilon \rho_{\eta}(\tau, \cdot)\right)^{2}\right) \rho_{\tau}(\tau, \cdot) \mathrm{d} \eta \\
&=\int_{-\ell_{0} / 2}^{\ell_{0} / 2} \mathcal{G}_{\varepsilon}\left(\left(\Phi_{\eta}(\tau, \cdot)\right)^{2}\right) \rho_{\tau}(\tau, \cdot) \mathrm{d} \eta+2 \varepsilon \int_{-\ell_{0} / 2}^{\ell_{0} / 2} \mathcal{G}_{\varepsilon}\left(\Phi_{\eta}(\tau, \cdot) \rho_{\eta}(\tau, \cdot)\right) \rho_{\tau}(\tau, \cdot) \mathrm{d} \eta \\
& \quad+\varepsilon^{2} \int_{-\ell_{0} / 2}^{\ell_{0} / 2} \mathcal{G}_{\varepsilon}\left(\left(\rho_{\eta}(\tau, \cdot)\right)^{2}\right) \rho_{\tau} \mathrm{d} \eta \\
&:= J_{1}(\tau)+J_{2}(\tau)+J_{3}(\tau) .
\end{aligned}
$$

To estimate $J_{1}$, we use (6.4) and the Hölder inequality to get

$$
\left|J_{1}(\tau)\right| \leqslant 2\left\|\rho_{\tau}(\tau, \cdot)\right\|_{2}\left\|\left(\left(\Phi_{\eta}(\tau, \cdot)\right)^{2}\right)_{\eta \eta}\right\|_{2} .
$$


Estimating the terms $J_{2}$ and $J_{3}$ is a bit more tricky. Using 6.5 and 6.6 , we get

$$
\begin{aligned}
\left|J_{2}(\tau)\right| \leqslant & 3 \varepsilon\left|\int_{-\ell_{0} / 2}^{\ell_{0} / 2} \rho_{\tau}(\tau, \cdot)\left(\Phi_{\eta}(\tau, \cdot) \rho_{\eta}(\tau, \cdot)\right)_{\eta \eta} \mathrm{d} \eta\right|+\frac{3}{2}\left\|\Phi_{\eta}(\tau, \cdot) \rho_{\eta}(\tau, \cdot)\right\|_{H^{1}}\left\|\rho_{\tau}(\tau, \cdot)\right\|_{2} \\
= & 3 \varepsilon\left|\int_{-\ell_{0} / 2}^{\ell_{0} / 2} \rho_{\tau \eta}(\tau, \cdot)\left(\Phi_{\eta \eta}(\tau, \cdot) \rho_{\eta}(\tau, \cdot)+\Phi_{\eta}(\tau, \cdot) \rho_{\eta \eta}(\tau, \cdot)\right) \mathrm{d} \eta\right| \\
& +\frac{3}{2}\left\|\Phi_{\eta}(\tau, \cdot) \rho_{\eta}(\tau, \cdot)\right\|_{H^{1}}\left\|\rho_{\tau}(\tau, \cdot)\right\|_{2} \\
\leqslant & 3 \varepsilon\left\|\Phi_{\eta \eta}\right\|_{\infty}\left\|\rho_{\tau \eta}(\tau, \cdot)\right\|_{2}\left\|\rho_{\eta}(\tau, \cdot)\right\|_{2}+3 \varepsilon\left\|\Phi_{\eta}\right\|_{\infty}\left\|\rho_{\tau \eta}(\tau, \cdot)\right\|_{2}\left\|\rho_{\eta \eta}(\tau, \cdot)\right\|_{2} \\
& +\frac{3}{2}\left\{\left(\left\|\Phi_{\eta}\right\|_{\infty}+\left\|\Phi_{\eta \eta}\right\|_{\infty}\right)\left\|\rho_{\eta}(\tau, \cdot)\right\|_{2}+\left\|\Phi_{\eta}\right\|_{\infty}\left\|\rho_{\eta \eta}(\tau, \cdot)\right\|_{2}\right\}\left\|_{\tau}(\tau, \cdot)\right\|_{2} \cdot
\end{aligned}
$$

Using the Poincaré-Wirtinger inequality, we can continue the estimate to obtain

$$
\left|J_{2}(\tau)\right| \leqslant C_{1}\left(\varepsilon\left\|\rho_{\tau \eta}(\tau, \cdot)\right\|_{2}\left\|\rho_{\eta \eta}(\tau, \cdot)\right\|_{2}+\left\|\rho_{\eta \eta}(\tau, \cdot)\right\|_{2}\left\|\rho_{\tau}(\tau, \cdot)\right\|_{2}\right)
$$

for some positive constant $C_{1}$, independent of $\tau, T^{\prime}$ and $\varepsilon$.

To estimate $J_{3}$ we argue similarly to get

$$
\begin{aligned}
\left|J_{3}(\tau)\right| \leqslant & \frac{3}{2} \varepsilon^{2}\left|\int_{-\ell_{0} / 2}^{\ell_{0} / 2} \rho_{\tau}(\tau, \cdot)\left(\left(\rho_{\eta}(\tau, \cdot)\right)^{2}\right)_{\eta \eta} \mathrm{d} \eta\right|+\frac{3}{2} \varepsilon\left\|\left(\rho_{\eta}(\tau, \cdot)\right)^{2}\right\|_{H^{1}}\left\|\rho_{\tau}(\tau, \cdot)\right\|_{2} \\
= & \frac{3}{2} \varepsilon^{2}\left|\int_{-\ell_{0} / 2}^{\ell_{0} / 2} \rho_{\tau \eta}(\tau, \cdot)\left(\left(\rho_{\eta}(\tau, \cdot)\right)^{2}\right)_{\eta} \mathrm{d} \eta\right|+\frac{3}{2} \varepsilon\left\|\left(\rho_{\eta}(\tau, \cdot)\right)^{2}\right\|_{H^{1}}\left\|\rho_{\tau}(\tau, \cdot)\right\|_{2} \\
\leqslant & 3 \varepsilon^{2}\left\|\rho_{\tau \eta}(\tau, \cdot)\right\|_{2}\left\|\rho_{\eta}(\tau, \cdot)\right\|_{\infty}\left\|\rho_{\eta \eta}(\tau, \cdot)\right\|_{2} \\
& +\frac{3}{2} \varepsilon\left\|\rho_{\eta}(\tau, \cdot)\right\|_{\infty}\left(\left\|\rho_{\eta}(\tau, \cdot)\right\|_{2}+2\left\|\rho_{\eta \eta}(\tau, \cdot)\right\|_{2}\right)\left\|\rho_{\tau}(\tau, \cdot)\right\|_{2} \\
\leqslant & C_{2}\left(\varepsilon^{2}\left\|\rho_{\tau \eta}(\tau, \cdot)\right\|_{2}\left\|\rho_{\eta \eta}(\tau, \cdot)\right\|_{2}^{2}+\varepsilon\left\|\rho_{\eta \eta}(\tau, \cdot)\right\|_{2}^{2}\left\|\rho_{\tau}(\tau, \cdot)\right\|_{2}\right)
\end{aligned}
$$

for some positive constant $C_{2}$, independent of $\tau, T^{\prime}$ and $\varepsilon$.

By combining 6.7, , 6.8) and 6.10, the assertion follows at once.

Lemma 6.5 There exists a positive constant $C$, independent of $\varepsilon \in(0,1 / 2], \tau$ and $T^{\prime}$, such that

$$
\left|\mathcal{J}_{4}(\tau)\right| \leqslant C\left(\left\|\Phi_{\tau \eta \eta}(\tau, \cdot)\right\|_{2}+\left\|\Phi_{\eta \eta \eta \eta}(\tau, \cdot)\right\|_{2}\right)\left\|\rho_{\tau}(\tau, \cdot)\right\|_{2}, \quad \tau \in\left[0, T^{\prime}\right] .
$$

Proof. Of course, we just need to estimate the terms

$$
\begin{aligned}
& J_{4}^{(1)}(\tau)=\varepsilon \int_{-\ell_{0} / 2}^{\ell_{0} / 2} \rho_{\tau}\left(D_{\eta \eta} R\left(0, L_{\varepsilon}\right)\left[\Phi_{\tau \eta \eta}\left(\mathbf{V}-\mathbf{T}-\frac{4}{3} \mathbf{U}\right)\right]\right)_{1}(\cdot, 0, \cdot) \mathrm{d} \eta, \\
& J_{4}^{(2)}(\tau)=\int_{-\ell_{0} / 2}^{\ell_{0} / 2} \rho_{\tau}\left(D_{\eta \eta} R\left(0, L_{\varepsilon}\right)\left[\Phi_{\eta \eta}\left(\mathbf{T}^{\prime}-\mathbf{U}\right)\right]\right)_{1}(\cdot, 0, \cdot) \mathrm{d} \eta ;
\end{aligned}
$$

the remaining terms are easily handled. 
Concerning $J_{4}^{(1)}$, taking 5.3 into account we can estimate

$$
\begin{aligned}
\left|J_{4}^{(1)}(\tau)\right| & \leqslant \frac{4}{9} \varepsilon \sum_{k=0}^{+\infty} \lambda_{k}^{2} \frac{4 X_{k}+7}{\left(X_{k}+1\right)^{2}\left(X_{k}+2\right)}\left|\widehat{\rho}_{\tau}(\tau, k)\right|\left|\widehat{\Phi}_{\tau}(\tau, k)\right| \\
& \leqslant \frac{1}{9} \sum_{k=0}^{+\infty} \lambda_{k} \frac{X_{k}^{2}\left(4 X_{k}+7\right)}{\left(X_{k}+1\right)^{2}\left(X_{k}+2\right)}\left|\widehat{\rho}_{\tau}(\tau, k)\right|\left|\widehat{\Phi}_{\tau}(\tau, k)\right| \\
& \leqslant \frac{4}{9} \sum_{k=0}^{+\infty} \lambda_{k}\left|\widehat{\rho}_{\tau}(\tau, k)\right|\left|\widehat{\Phi}_{\tau}(\tau, k)\right|=\frac{4}{9}\left\|\rho_{\tau}(\tau, \cdot)\right\|_{2}\left\|\Phi_{\tau \eta \eta}(\tau, \cdot)\right\|_{2}
\end{aligned}
$$

for any $\tau \in\left[0, T^{\prime}\right]$. Similarly, using 5.7 we can estimate

$$
\begin{aligned}
\left|J_{4}^{(2)}(\tau)\right| & \leqslant \frac{2}{3} \sum_{k=0}^{+\infty} \lambda_{k}^{2} \frac{1}{\left(X_{k}+1\right)\left(X_{k}+2\right)}\left|\widehat{\rho}_{\tau}(\tau, k)\right||\widehat{\Phi}(\tau, k)| \leqslant \frac{1}{3} \sum_{k=0}^{+\infty} \lambda_{k}^{2}\left|\widehat{\rho}_{\tau}(\tau, k)\right||\widehat{\Phi}(\tau, k)| \\
& =\frac{1}{3}\left\|\rho_{\tau}(\tau, \cdot)\right\|_{2}\left\|\Phi_{\eta \eta \eta \eta}(\tau, \cdot)\right\|_{2}
\end{aligned}
$$

for any $\tau \in\left[0, T^{\prime}\right]$. Now, estimate 6.11 follows immediately.

Finally, we recall the following technical result proved in [1], which plays a crucial role in the proof of Theorem 6.1.

Lemma 6.6 (slight extension of [1, Lemma 3.1]) Let $A_{0}, C_{0}, C_{1}, C_{2}$ be positive constants. For any $T>0$, there exist $\varepsilon_{0} \in(0,1 / 2)$ and a constant $K_{0}$ such that, if $A_{\varepsilon} \in C^{1}\left(\left[0, T^{\prime}\right]\right)\left(T^{\prime} \in(0, T]\right)$ satisfies

$$
\left\{\begin{array}{l}
A_{\varepsilon}^{\prime}(\tau) \leqslant C_{0}+C_{1} A_{\varepsilon}(\tau)+C_{2} \varepsilon\left(A_{\varepsilon}(\tau)\right)^{2}, \quad \tau \in\left[0, T^{\prime}\right] \\
A_{\varepsilon}(0) \leqslant A_{0}
\end{array}\right.
$$

for some $\varepsilon \in\left(0, \varepsilon_{0}\right]$, then $A_{\varepsilon}(\tau) \leqslant K_{0}$ for any $\tau \in\left[0, T^{\prime}\right]$.

Proof of Theorem 6.1 To begin, we observe that, invoking the Poincaré-Wirtinger inequality, we can estimate

$$
\left|\int_{-\ell_{0} / 2}^{\ell_{0} / 2}\left(\rho_{\eta}(\tau, \cdot)\right)^{2} \rho_{\tau}(\tau, \cdot) \mathrm{d} \eta\right| \leqslant\left\|\rho_{\tau}(\tau, \cdot)\right\|_{2}\left\|\rho_{\eta}(\tau, \cdot)\right\|_{2}\left\|\rho_{\eta}(\tau, \cdot)\right\|_{\infty} \leqslant \sqrt{\ell_{0}}\left\|\rho_{\tau}(\tau, \cdot)\right\|_{2}\left\|\rho_{\eta \eta}(\tau, \cdot)\right\|_{2}^{2}
$$

for any $\tau \in\left[0, T^{\prime}\right]$. Hence, Lemmata 6.2 6.5. estimate $(6.3)$ and the Hölder inequality give

$$
\begin{aligned}
& \frac{3}{2}(1-\varepsilon) \frac{\mathrm{d}}{\mathrm{d} \tau}\left\|\rho_{\eta \eta}(\tau, \cdot)\right\|_{2}^{2}+\left\|\rho_{\tau}(\tau, \cdot)\right\|_{2}^{2}+3 \varepsilon\left\|\rho_{\tau \eta}(\tau, \cdot)\right\|_{2}^{2} \\
& \leqslant K\left(\left\|\rho_{\tau}(\tau, \cdot)\right\|_{2}+\left\|\rho_{\tau}(\tau, \cdot)\right\|_{2}\left\|\rho_{\eta \eta}(\tau, \cdot)\right\|_{2}+\varepsilon\left\|\rho_{\tau}(\tau, \cdot)\right\|_{2}\left\|\rho_{\eta \eta}(\tau, \cdot)\right\|_{2}^{2}\right. \\
&\left.+\varepsilon\left\|\rho_{\tau \eta}(\tau, \cdot)\right\|_{2}\left\|\rho_{\eta \eta}(\tau, \cdot)\right\|_{2}+\varepsilon^{2}\left\|\rho_{\tau \eta}(\tau, \cdot)\right\|_{2}\left\|\rho_{\eta \eta}(\tau, \cdot)\right\|_{2}^{2}\right)
\end{aligned}
$$


for some positive constant $K$, depending on $\Phi$ but independent of $\tau \in\left[0, T^{\prime}\right]$. Using the Young inequality $a b \leqslant \frac{1}{4} a^{2}+b^{2}$ we get

$$
\begin{aligned}
& \frac{3}{2}(1-\varepsilon) \frac{\mathrm{d}}{\mathrm{d} \tau}\left\|\rho_{\eta \eta}(\tau, \cdot)\right\|_{2}^{2}+\left\|\rho_{\tau}(\tau, \cdot)\right\|_{2}^{2}+3 \varepsilon\left\|\rho_{\tau \eta}(\tau, \cdot)\right\|_{2}^{2} \\
& \leqslant K^{2}+\frac{3}{4}\left\|\rho_{\tau}(\tau, \cdot)\right\|_{2}^{2}+\frac{1}{4}\left(\varepsilon+\varepsilon^{2}\right)\left\|\rho_{\tau \eta}(\tau, \cdot)\right\|_{2}^{2} \\
&+K^{2}\left(1+\varepsilon+\varepsilon^{2}\right)\left\|\rho_{\eta \eta}(\tau, \cdot)\right\|_{2}^{2}+K^{2} \varepsilon^{2}\left\|\rho_{\eta \eta}(\tau, \cdot)\right\|_{2}^{4},
\end{aligned}
$$

or, equivalently,

$$
\begin{aligned}
\frac{\mathrm{d}}{\mathrm{d} \tau}\left\|\rho_{\eta \eta}(\tau, \cdot)\right\|_{2}^{2} & \leqslant \frac{4}{3} K^{2}+4 K^{2}\left\|\rho_{\eta \eta}(\tau, \cdot)\right\|_{2}^{2}+\frac{4}{3} K^{2} \varepsilon^{2}\left\|\rho_{\eta \eta}(\tau, \cdot)\right\|_{2}^{4} \\
& \leqslant \frac{4}{3} K^{2}+4 K^{2}\left\|\rho_{\eta \eta}(\tau, \cdot)\right\|_{2}^{2}+\frac{2}{3} K^{2} \varepsilon\left\|\rho_{\eta \eta}(\tau, \cdot)\right\|_{2}^{4}
\end{aligned}
$$

provided that $\varepsilon \leqslant 1 / 2$. Applying Lemma 6.6 to 6.14 with $\left(C_{0}, C_{1}, C_{2}\right)=\left(4 K^{2} / 3,4 K^{2}, 2 K^{2} / 3\right)$ and $A_{\varepsilon}(\tau)=\left\|\rho_{\eta \eta}(\tau, \cdot)\right\|_{2}^{2}$, we deduce that there exist $\varepsilon_{0} \in(0,1 / 2)$ and $K_{0}>0$ such that

$$
\sup _{\tau \in\left(0, T^{\prime}\right]} \int_{-\ell_{0} / 2}^{\ell_{0} / 2}\left(\rho_{\eta \eta}(\tau, \eta)\right)^{2} \mathrm{~d} \eta \leqslant K_{0}
$$

for any $\varepsilon \in\left(0, \varepsilon_{0}\right)$. Now, using again the Poincaré-Wirtinger inequality, we get

$$
\sup _{\substack{\tau \in\left(0, T^{\prime}\right] \\ \eta \in\left[-\ell_{0} / 2, \ell_{0} / 2\right]}}\left|\rho_{\eta}(\tau, \eta)\right| \leqslant K_{1}
$$

for any $\varepsilon \in\left(0, \varepsilon_{0}\right]$, with $K_{1}$ independent of $\varepsilon$ and $T^{\prime}<T$.

Finally, integrating 6.13 and using the estimates obtained so far, we deduce that

$$
\int_{0}^{T^{\prime}} \int_{-\ell_{0} / 2}^{\ell_{0} / 2}\left(\rho_{\tau}(\tau, \eta)\right)^{2} \mathrm{~d} \tau \mathrm{d} \eta+\int_{0}^{T^{\prime}} \int_{-\ell_{0} / 2}^{\ell_{0} / 2}\left(\rho_{\tau \eta}(\tau, \eta)\right)^{2} \mathrm{~d} \tau \mathrm{d} \eta \leqslant K_{2}
$$

for some constant $K_{2}$, independent of $\varepsilon$ and $T^{\prime}<T$. The assertion now follows.

COROLlary 6.7 Under the assumptions of Theorem 6.1, there exists a constant $M>0$, independent of $T^{\prime}<T$, such that

$$
\|\rho\|_{C^{0,1}\left(\left[0, T^{\prime}\right] \times\left[-\ell_{0} / 2, \ell_{0} / 2\right]\right)} \leqslant M
$$

for any $\varepsilon \in\left(0, \varepsilon_{0}\right]$.

Proof. In view of Theorem 6.1 we only have to estimate the sup-norm of the function $\rho$, but this is immediate if we observe that 6.15 implies that $\rho$ is bounded in $H^{1}\left(\left[0, T^{\prime}\right] ; H^{1}\right)$ by a constant independent of $T^{\prime}$, and $\rho(0, \cdot)=0$. 
6.2 Solving equation (4.20) in $[0, T]$

We now consider a fixed time interval $[0, T]$ and $0<\varepsilon \leqslant \varepsilon_{0}$, with $\varepsilon_{0}=\varepsilon_{0}(T)$ given by Theorem 6.1. Thanks to the a priori estimates of Subsection 6.1 and a classical result for semilinear problems, we can show that, for any $\varepsilon \in\left(0, \varepsilon_{0}\right]$, the solution $\rho=\rho_{\varepsilon}$ to problem 4.20$)$, given by Theorem 5.1. can be extended to a function $\rho \in y_{T}$ (see Definition 4.1) which solves the equation in the whole of $[0, T]$.

Theorem 6.8 Fix $T>0$ and let $\varepsilon_{0}=\varepsilon_{0}(T)$ be as in Theorem 6.1. Then, for any $\varepsilon \in\left(0, \varepsilon_{0}\right]$, equation 4.20) admits a unique solution $\rho \in y_{T}$.

Proof. Let us fix $T$ as in the statement of the theorem and let $\varepsilon \in\left(0, \varepsilon_{0}\right)$. Suppose for contradiction that $T_{\varepsilon}<T$. Then, by Theorem 6.1 applied with any $T^{\prime}<T_{\varepsilon}$, we get

$$
\sup _{\tau \in\left[0, T_{\varepsilon}\right)}\|\rho(\tau, \cdot)\|_{C^{1}}<K
$$

for some positive constant $K$, independent of $\varepsilon$. Hence, the function $\mathcal{K}_{\varepsilon}\left(\cdot, \rho_{\eta}\right)$ is bounded in $\left[0, T_{\varepsilon}\right) \times\left[-\ell_{0} / 2, \ell_{0} / 2\right]$. In view of [11, Prop. 7.1.8], applied to equation (5.5), and the bootstrap argument in the proof of Theorem 5.1, this leads us to a contradiction.

\subsection{Proof of Main Theorem 1.1}

We are now in a position to prove the main result of this paper. Let us fix a function $\Phi_{0} \in C_{\sharp}^{4+4 \beta}$ for some $\beta \in(1 / 2,1)$.

From the results in Subsection 6.2 we know that, for any $T>0$, there exists $\varepsilon_{0}=\varepsilon_{0}(T)$ such that equation (4.16) admits a unique solution $\psi_{\varepsilon} \in y_{T}$ such that $\rho(0, \cdot)=\Phi_{0}$. Moreover, by Corollary 6.7

$$
\left\|\psi_{\varepsilon}(\tau, \cdot)-\Phi_{0}(\tau, \cdot)\right\|_{C^{0}} \leqslant \varepsilon M, \quad \tau \in[0, T]
$$

for some positive constant $M$ and any $\varepsilon \in\left(0, \varepsilon_{0}\right]$.

In view of Theorem 4.3 there exists a function $v \in \mathcal{V}_{T}$ such that the pair $(v, \psi)$ is the unique solution to problem $3.1-(3.3)$.

Coming back to problem (1.8)-(1.10) and setting $\ell_{\varepsilon}=\ell_{0} / \sqrt{\varepsilon}$ and $T_{\varepsilon}=T / \varepsilon^{2}$, it is now immediate to conclude that, for any $\varepsilon \in\left(0, \varepsilon_{0}\right]$, it admits a unique solution $(u, \varphi) \in \mathcal{V}_{T_{\varepsilon}} \times y_{T_{\varepsilon}}$. Moreover,

$$
\left\|\varphi_{\varepsilon}(t, \cdot)-\varepsilon \Phi\left(t \varepsilon^{2}, \sqrt{\varepsilon} \cdot\right)\right\|_{C\left(\left[-\ell_{\varepsilon} / 2, \ell_{\varepsilon} / 2\right]\right)} \leqslant \varepsilon^{2} M, \quad t \in\left[0, T_{\varepsilon}\right] .
$$

This accomplishes the proof of the Main Theorem.

\section{A. Some results from [4]}

In this appendix we recall some results from [4] that are used throughout this paper.

\section{A.1 The operator $L$}

Let $\mathcal{L}$ be the differential operator defined on smooth functions $\mathbf{u}$ by

$$
(\mathcal{L} \mathbf{u})(x, \eta)= \begin{cases}D_{x x} u_{1}(x, \eta)-D_{x} u_{1}(x, \eta)+e^{x} u_{1}(0, \eta), & x \leqslant 0,|\eta| \leqslant \ell_{0} / 2, \\ D_{x x} u_{2}(x, \eta)-D_{x} u_{2}(x, \eta), & x \geqslant 0,|\eta| \leqslant \ell_{0} / 2,\end{cases}
$$


and let $L$ be its realization in $\mathcal{X}$, defined by

$$
\left\{\begin{array}{l}
D(L)=\left\{\mathbf{u} \in C^{2,0}\left(I_{-}\right) \times C^{2,0}\left(I_{+}\right): \mathbf{u},\right. \\
\left.L \mathbf{L} \mathbf{u} \in X, D_{x}^{(j)} u_{1}(0, \cdot)=D_{x}^{(j)} u_{2}(0, \cdot), j=0,1\right\}, \\
L \mathbf{u}= \begin{cases}D_{x x} u_{1}-D_{x} u_{1}+e^{x} u_{1}(0, \cdot), & (x, \eta) \in I_{-}, \\
D_{x x} u_{2}-D_{x} u_{2}, & (x, \eta) \in I_{+} .\end{cases}
\end{array}\right.
$$

THEOREM A.1 The following properties are satisfied:

(i) the operator $L$ is sectorial, and hence it generates an analytic semigroup in $X$;

(ii) the spectrum of the operator $L$ consists of 0 and the halfline $(-\infty,-1 / 4]$;

(iii) the spectral projection on the kernel of $L$ is the operator $\mathcal{P}$ defined by

$$
\mathcal{P}(\mathbf{f})=\left(\int_{-\infty}^{0} f_{1}(x, \cdot) \mathrm{d} x+\int_{0}^{+\infty} e^{-x} f_{2}(x, \cdot) \mathrm{d} x\right) \mathbf{U}=Q(\mathbf{f}) \mathbf{U}, \quad \mathbf{f} \in X
$$

(iv) let $\mathbf{f} \in \mathcal{X}$; then the equation $L \mathbf{u}=\mathbf{f}$ has a solution $\mathbf{u} \in D(L)$ if and only if $\mathcal{P}(\mathbf{f})=0$;

(v) for any $\lambda \notin(-\infty,-1 / 4] \cup\{0\}$ and any $\mathbf{f}=\left(f_{1}, f_{2}\right) \in \mathcal{X}$, setting $\mathbf{u}:=R(\lambda, L) \mathbf{f}$ we have

$$
u_{1}(0, \eta)=u_{2}(0, \eta)=g(\lambda)\left(\int_{-\infty}^{0} e^{-v_{1} t} f_{1}(t, \eta) \mathrm{d} t+\int_{0}^{+\infty} e^{-v_{2} t} f_{2}(t, \eta) \mathrm{d} t\right)
$$

for any $\eta \in\left[-\ell_{0} / 2, \ell_{0} / 2\right]$, where

$$
g(\lambda)=\left(\frac{2 \lambda}{1+(2 \lambda-1) X(\lambda)} \frac{1}{\nu_{2}}+1\right) \frac{1}{X(\lambda)}, \quad X(\lambda)=\sqrt{1+4 \lambda} .
$$

\section{A.2 The operator $L_{\varepsilon}$}

For any $\varepsilon>0$, we consider the operator $L+\varepsilon A$ defined by

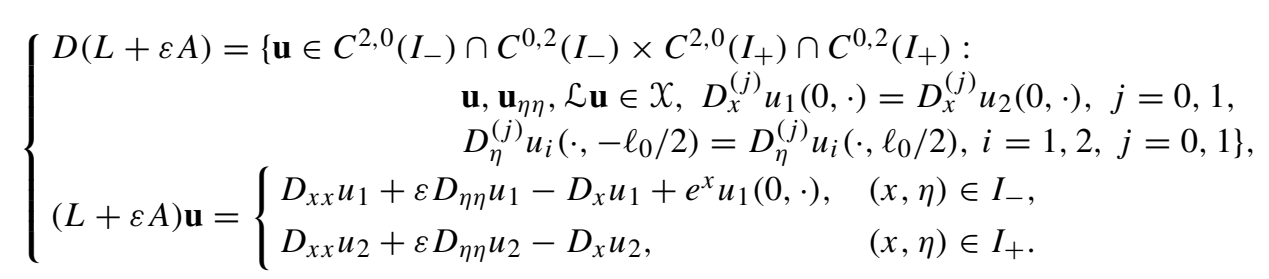

THEOREM A.2 The following properties are satisfied:

(i) the operator $L+\varepsilon A$ is closable and its closure $L_{\varepsilon}$ is sectorial;

(ii) the restriction of $L_{\varepsilon}$ to $(I-\mathcal{P})(\mathcal{X})$ is sectorial and 0 is in its resolvent set;

(iii) let $\mathbf{f}=\mathbf{h} \varphi$ for some $\mathbf{h} \in(I-\mathcal{P})(X)$, independent of $\eta$, and some $\varphi \in C_{\sharp}^{2 \alpha}(\alpha \in(0,1) \backslash\{1 / 2\})$; then the function $R\left(0, L_{\varepsilon}\right) \mathbf{f}$ belongs to $D(L+\varepsilon A)$, and there exists a positive constant $C$, depending on $\varepsilon$ and $\alpha$ but independent of $\mathbf{h}$ and $\varphi$, such that

$$
\left\|D_{x}^{(i)} R\left(0, L_{\varepsilon}\right) \mathbf{f}\right\| x+\left\|D_{\eta}^{(i)} R\left(0, L_{\varepsilon}\right) \mathbf{f}\right\| x \leqslant C\|\mathbf{h}\| x\|\varphi\|_{C^{2 \alpha}}
$$

for $i=0,1,2$. 


\section{A.3 Proof of Theorem 4.4}

We split the proof into three steps. In the first one, we show that problem (4.19) admits a unique solution $\Phi$ in some time domain $[0, T)$. Since this result can be proved using the same arguments as in Subsection 5.3, we just sketch the proof. Then, in Steps 2 and 3, we show that $\Phi$ exists and is smooth in the whole of $[0,+\infty)$.

Step 1. By the proof of Proposition 5.4 the realization $A$ of the second-order derivative in $C^{0}$, with domain $C_{\sharp}^{1} \cap C^{2}$, is a sectorial operator with spectrum contained in $(-\infty, 0]$. Hence, [11] Prop. 2.4.1 $\&$ 2.4.4] applies and shows that the operator $B:=-3 A^{2}-A$ is sectorial in $C^{0}$ with domain $D\left(A^{2}\right)$. Moreover, $D_{B}(\alpha, \infty)=C_{\sharp}^{4 \alpha}$, with equivalence of norms, for any $\alpha \in(0,2)$ such that $4 \alpha \notin \mathbb{N}$.

The variation of constants formula shows that any solution $\Phi \in C^{1,4}\left([0,+\infty) \times\left[-\ell_{0} / 2, \ell_{0} / 2\right]\right)$ to the Cauchy problem 4.19) is a fixed point of the operator $\Gamma$, formally defined by

$$
(\Gamma(\Phi))(\tau, \cdot)=e^{\tau B} \Phi_{0}+\int_{0}^{\tau} e^{(\tau-s) B}\left(\Phi_{\eta}(s, \cdot)\right)^{2} \mathrm{~d} s, \quad \tau>0,
$$

where $\left\{e^{t B}\right\}$ denotes the semigroup generated by $B$.

Let us fix $\alpha \in(1 / 4,1 / 2)$. Theorem 7.1.2 in [11] implies that $\Gamma$ has a unique fixed point $\Phi$ in $C\left(\left[0, T_{0}\right] ; D_{B}(\alpha, \infty)\right)$. A bootstrap argument allows us to prove that $\Phi$ belongs to $y_{T_{0}}$. Using [11, Prop. 4.2.1] and our assumptions on $\Phi_{0}$, it can be shown first that $\Phi \in C^{\beta, 4 \gamma}\left(\left[0, T_{0}\right] \times\right.$ $\left.\left[-\ell_{0} / 2, \ell_{0} / 2\right]\right)$ for any $\beta, \gamma \in(0,1)$, and then that $\Phi_{\eta} \in C^{\beta}\left(\left[0, T_{0}\right] \times\left[-\ell_{0} / 2, \ell_{0} / 2\right]\right)$ for any $\beta \in(0,3 / 4)$. Moreover, $D_{\eta}^{(j)} \Phi\left(\cdot,-\ell_{0} / 2\right) \equiv D_{\eta}^{(j)} \Phi\left(\cdot, \ell_{0} / 2\right)$ for $j=0,1,2,3$. Next, applying [11, Thm. 4.3.1(i)], we deduce that $\Phi \in C^{1,4}\left([0, T] \times\left[-\ell_{0} / 2, \ell_{0} / 2\right]\right)$ and is a solution to problem (4.19). Moreover, since $\Phi_{0} \in D_{B}(3 / 2+\alpha / 4, \infty), \Phi_{\tau}$ is bounded in $\left[0, T_{0}\right]$ with values in $D_{B}(1 / 2+\alpha / 4, \infty)$. Hence, $\Phi_{\tau} \in C^{0,2+\alpha}\left(\left[0, T_{0}\right] \times\left[-\ell_{0} / 2, \ell_{0} / 2\right]\right)$. As a byproduct, $\Phi_{\eta \eta \eta \eta} \in C^{0,2+\alpha}\left(\left[0, T_{0}\right] \times\left[-\ell_{0} / 2, \ell_{0} / 2\right]\right)$ and $D_{\eta}^{(j)} \Phi\left(\cdot,-\ell_{0} / 2\right)=D_{\eta}^{(j)} \Phi\left(\cdot, \ell_{0} / 2\right)$ for $j=4,5,6$.

Using a continuation argument, we can extend $\Phi$ to a maximal domain $[0, T)$ with a function (still denoted by $\Phi$ ) which belongs to $y_{T^{\prime}}$ for any $T^{\prime}<T$.

The rest of the proof is devoted to showing that $T=+\infty$. The main step is an a priori estimate suggested by the proof [13, Thm. 2.4], which deals with $L^{2}$-regularity for the K-S equation.

Step 2. Here we show that

$$
\left\|\Phi_{\eta}(\tau, \cdot)\right\|_{2} \leqslant e^{13 \tau / 6}\left\|D_{\eta} \Phi_{0}\right\|_{2}, \quad \tau \in[0, T) .
$$

For this purpose, we introduce the function $v$ defined by $v(\tau, \eta)=e^{-2 \tau} \Phi_{\eta}(\tau, \eta)$ for any $(\tau, \eta) \in$ $[0, T) \times\left[-\ell_{0} / 2, \ell_{0} / 2\right]$. The smoothness of $\Phi$ implies that $v \in C^{1,4}\left([0, T) \times\left[-\ell_{0} / 2, \ell_{0} / 2\right]\right)$ solves the parabolic equation

$$
v_{\tau}=-3 v_{\eta \eta \eta \eta}-v_{\eta \eta}-e^{2 \tau} v v_{\eta}-2 v,
$$

and satisfies the boundary conditions $D_{\eta}^{(k)} v\left(\tau,-\ell_{0} / 2\right)=D_{\eta}^{(k)} v\left(\tau, \ell_{0} / 2\right)$ for any $\tau \in[0, T)$ and $k=0,1,2,3$. Multiplying both sides of A4 by $v(\tau, \cdot)$, integrating on $\left(-\ell_{0} / 2, \ell_{0} / 2\right)$ and observing that the integral over $\left(-\ell_{0} / 2, \ell_{0} / 2\right)$ of $(v(\tau, \cdot))^{2} v_{\eta}(\tau, \cdot)$ vanishes for any $\tau \in(0, T]$, we get

$$
\frac{\mathrm{d}}{\mathrm{d} \tau}\|v(\tau, \cdot)\|_{2}^{2}+3\left\|v_{\eta \eta}(\tau, \cdot)\right\|_{2}^{2}-\left\|v_{\eta}(\tau, \cdot)\right\|_{2}^{2}+2\|v(\tau, \cdot)\|_{2}^{2}=0, \quad \tau \in[0, T) .
$$


In view of the estimate

$$
\left\|v_{\eta}(\tau, \cdot)\right\|_{2}^{2} \leqslant\|v(\tau, \cdot)\|_{2}\left\|v_{\eta \eta}(\tau, \cdot)\right\|_{2} \leqslant 3\left\|v_{\eta \eta}(\tau, \cdot)\right\|_{2}^{2}+\frac{5}{3}\|v(\tau, \cdot)\|_{2}^{2}, \quad \tau \in[0, T),
$$

formula $\mathrm{A} 5$ leads us to the inequality

$$
\frac{\mathrm{d}}{\mathrm{d} \tau}\|v(\tau, \cdot)\|_{2}^{2}+\frac{1}{3}\|v(\tau, \cdot)\|_{2}^{2} \leqslant 0, \quad \tau \in[0, T),
$$

from which estimate $\mathrm{A} 3$ ) follows at once.

Step 3. Let us consider the function $\Psi$ defined by $\Psi(\tau, \eta)=\Phi(\tau, \eta)-\Pi(\Phi(\tau, \cdot))$ for any $\tau \in$ $[0, T)$ and any $\eta \in\left[-\ell_{0} / 2, \ell_{0} / 2\right]$, where $\Pi(\Phi(\tau, \cdot))$ denotes the average of $\Phi(\tau, \cdot)$ over the interval $\left(-\ell_{0} / 2, \ell_{0} / 2\right)$. Applying the Poincaré-Wirtinger inequality, we get

$$
\|\Phi(\tau, \cdot)-\Pi(\Phi(\tau, \cdot))\|_{\infty} \leqslant \sqrt{\ell_{0}} e^{13 \tau / 6}\left\|D_{\eta} \Phi_{0}\right\|_{2}, \quad \tau \in[0, T) .
$$

Let us now show that the function $\tau \mapsto \Pi(\Phi(\tau, \cdot))$ satisfies a similar estimate. For this purpose, we fix $T^{\prime} \in(0, T), \tau \in\left[0, T^{\prime}\right)$, and apply the operator $\Pi$ to both sides of 4.19]. Since $\Phi$ and its derivatives satisfy periodic boundary conditions,

$$
\frac{\mathrm{d}}{\mathrm{d} \tau} \Pi(\Phi(\tau, \cdot))=\Pi\left(\Phi_{\tau}(\tau, \cdot)\right)=-\frac{1}{2 \ell_{0}} \Pi\left(\left(\Phi_{\eta}(\tau, \cdot)\right)^{2}\right)
$$

for any $\tau \in[0, T)$. Taking $\mathrm{A} 3]$ into account, we can then estimate

$$
\left|\frac{\mathrm{d}}{\mathrm{d} \tau} \Pi(\Phi(\tau, \cdot))\right| \leqslant \frac{1}{2 \ell_{0}} e^{13 \tau / 3}\left\|D_{\eta} \Phi_{0}\right\|_{2}^{2}, \quad \tau \in[0, T) .
$$

Hence,

$$
|\Pi(\Phi(\tau))| \leqslant\left|\Pi\left(\Phi_{0}\right)\right|+\int_{0}^{\tau}\left|\frac{\mathrm{d}}{\mathrm{d} \tau} \Pi(\Phi(\tau, \cdot))\right| \mathrm{d} \tau \leqslant\left|\Pi\left(\Phi_{0}\right)\right|+\frac{3}{26 \ell_{0}}\left\|D_{\eta} \Phi_{0}\right\|_{2}^{2} e^{13 \tau / 3}
$$

for any $\tau \in[0, T)$. Estimates A6 and (A7) show that $\Phi$ is bounded in $[0, T) \times\left[-\ell_{0} / 2, \ell_{0} / 2\right]$. Therefore, we can apply [11, Prop. 7.2.2] with $X_{\alpha}=D_{B}(\alpha, \infty)$, which implies that $T=+\infty$.

\section{Acknowledgments}

J.H. and L.L. were visiting professors at the University of Bordeaux 1 respectively in 2006-2007 and 2007-2009. They gratefully acknowledge the warm hospitality of the Institute of Mathematics of Bordeaux during their visits.

We wish to thank the referees for their comments which allowed us to improve the quality of the paper.

\section{REFERENCES}

[1] Brauner, C.-M., Frankel, M. L., Hulshof, J., Lunardi, A., \& Sivashinsky, G. I. On the $\kappa-\theta$ model of cellular flames: existence in the large and asymptotics. Discrete Contin. Dynam. Systems 1 (2007), 27-39. Zbl 1152.35010 MR 2375579 
[2] Brauner, C.-M., Frankel, M. L., Hulshof, J., \& Roytburd, V. Stability and attractors for quasi-steady model of cellular flames. Interfaces Free Bound. 8 (2006), 301-316. Zbl 1109.35097 MR 2273231

[3] Brauner, C.-M., Frankel, M. L., Hulshof, J., \& Sivashinsky, G. I. Weakly nonlinear asymptotic of the $\kappa-\theta$ model of cellular flames: the Q-S equation. Interfaces Free Bound. 7 (2005), 131-146. Zbl 1087.35092 MR 2148913

[4] Brauner, C.-M., Hulshof, J., \& Lorenzi, L. Stability of the travelling wave in a 2D weakly nonlinear Stefan problem. Kinet. Relat. Models 2 (2009), 109-134. Zbl 1185.35289 MR 2472151

[5] Brauner, C.-M., Hulshof, J., Lorenzi, L., \& Sivashinsky, G. I. A fully nonlinear equation for the flame front in a quasi-steady combustion model. Discrete Contin. Dynam. Systems 27 (2010), 1415-1446. Zbl 1196.35122 MR 2629531

[6] Brauner, C.-M., Lorenzi, L., Sivashinsky, G. I., \& XU, C.-J. On a strongly damped wave equation for the flame front. Chinese Ann. Math. 31 (2010), 819-840. Zbl pre05841064

[7] Eckhaus, W. Asymptotic Analysis of Singular Perturbations. North-Holland (1979). Zbl 0421.34057 MR 0553107

[8] Frankel, M. L. On the weakly nonlinear evolution of a perturbed solid-liquid interface. Phys. D 27 (1987), 260-266. Zbl 0663.35093

[9] Frankel, M. L., Roytburd, V., \& Sivashinsky, G. I. Complex dynamics generated by a sharp interface model of self-propagating high-temperature synthesis. Combust. Theory Modelling 2 (1998), 479-496. Zbl 0934.80003

[10] Hyman, J. M., \& Nicolaenko, B. The Kuramoto-Sivashinsky equation: a bridge between PDEs and dynamical systems, Phys. D 18 (1986), 113-126. Zbl 0602.58033 MR 0838315

[11] Lunardi, A. Analytic Semigroups and Optimal Regularity in Parabolic Problems. Birkhäuser, Basel (1995). Zbl 0816.35001 MR 1329547

[12] Sivashinsky, G. I. On flame propagation under conditions of stoichiometry. SIAM J. Appl. Math. 39 (1980), 67-82. Zbl 0464.76055 MR 0585829

[13] TADMOR, E. The well-posedness of the Kuramoto-Sivashinsky equation. SIAM J. Math. Anal. 17 (1986), 884-893. Zbl 0606.35073 MR 0846395

[14] Temam, R. Infinite-Dimensional Dynamical Systems in Mechanics and Physics. 2nd ed., Appl. Math. Sci. 68, Springer (1997). Zbl 0871.35001 MR 1441312

[15] Triebel, H. Interpolation Theory, Function Spaces, Differential Operators. North-Holland, Amsterdam (1978). Zbl 0387.46032 MR 0503903 